\title{
A local characterization of affine holomorphic immersions with an anti-complex and $\nabla$-parallel shape operator
}

\author{
by Maria Robaszewska (Kraków)
}

\begin{abstract}
We study the complex hypersurfaces $f: M^{(n)} \rightarrow \mathbb{C}^{n+1}$ which together with their transversal bundles have the property that around any point of $M$ there exists a local section of the transversal bundle inducing a $\nabla$-parallel anti-complex shape operator $S$. We give a class of examples of such hypersurfaces with an arbitrary rank of $S$ from 1 to $[n / 2]$ and show that every such hypersurface with positive type number and $S \neq 0$ is locally of this kind, modulo an affine isomorphism of $\mathbb{C}^{n+1}$.
\end{abstract}

1. Introduction. Among the connections induced on complex hypersurfaces $f: M^{(n)} \rightarrow \mathbb{C}^{n+1}$ by $\mathcal{C}^{\infty}$ complex transversal bundles there are two particular kinds of great interest: holomorphic connections and affine Kähler connections. The latter are meant to be a generalization of Kähler connections. In terms of the curvature tensor, a holomorphic affine connection is characterized by the condition

$$
R(J X, Y)=J R(X, Y) \quad \text { for all vector fields } X, Y,
$$

while for an affine Kähler connection we have, by definition,

$$
R(J X, J Y)=R(X, Y) \quad \text { for all } X, Y
$$

(see $[\mathrm{NS}]$ ). Since, provided the affine fundamental form $h$ does not vanish on $M$, a holomorphic connection is induced by a holomorphic transversal bundle, it is possible to adapt the ideas from the real affine hypersurface geometry to this case. For instance, having a non-degenerate hypersurface one can construct a holomorphic analogue of affine normal vector field [DVV]. Then one can consider the condition $S=\lambda I, \lambda=$ const, which describes the affine spheres [DVV].

On the contrary, the non-flat affine Kähler connections induced on hypersurfaces (in particular, the non-flat Kähler ones) cannot be treated in this

2000 Mathematics Subject Classification: 53A15, 53B05, 53C56.

Key words and phrases: affine holomorphic immersion, affine Kähler connection, anticomplex shape operator, parallel shape operator. 
way. Instead of the holomorphic transversal bundles, with the complex shape operator, one has to consider the transversal bundles $\mathcal{N}$ having the property that the shape operator corresponding to sections of $\mathcal{N}$ is anti-complex. This property of $\mathcal{N}$ implies the desired condition for the curvature tensor $R$ of $\nabla$ and is necessary for $\nabla$ to be affine Kähler if $t f>1$ at some point of $M$ (see $[\mathrm{O}]$ ).

Clearly, no section $\xi$ of such a bundle can induce $S$ proportional to the identity, except for the case $S=0$. Being $\nabla$-parallel is a weaker condition on $S$ than $S=\lambda I, \lambda=$ const. This condition is shown to have some non-trivial realizations even if we require $S$ to be anti-complex. It is worth noting that we need to consider degenerate hypersurfaces, because the nondegeneracy implies $S=0$ (Lemma 2).

2. Preliminaries. Let $M$ be an $n$-dimensional connected complex manifold. We shall consider a holomorphic immersion $f: M \rightarrow \mathbb{C}^{n+1}$ together with a $\mathcal{C}^{\infty}$ complex transversal bundle $\mathcal{N}$. If $\xi: U \rightarrow \mathbb{C}^{n+1}$ is a local section of $\mathcal{N}$, then the induced connection $\nabla$ on $M$, the second fundamental form $h$, the shape operator $S$ and the transversal forms $\mu$ and $\nu$ are defined by the following Gauss and Weingarten formulas [NS]:

$$
\begin{aligned}
D_{X} f_{*} Y & =f_{*} \nabla_{X} Y+h(X, Y) \xi-h(J X, Y) J \xi, \\
D_{X} \xi & =-f_{*} S X+\mu(X) \xi+\nu(X) J \xi .
\end{aligned}
$$

Here $D$ denotes the standard connection on $\mathbb{C}^{n+1}$, and $J$ the complex structure on $M$ and on $\mathbb{C}^{n+1}$ as well.

Let $m \in M$. The complex rank of the $\mathbb{C}$-bilinear form $h_{m}^{c}(\cdot, \cdot)=h_{m}(\cdot, \cdot)-$ $i h_{m}(J \cdot, \cdot)$ depends on $f$ only. It is called the type number of $f$ at $m$ and denoted by $t f_{m}$ (see $[\mathrm{O}]$ ). We shall assume that it is positive everywhere on $M$.

Our first requirement on the transversal bundle is that the induced shape operator $S$ is anti-complex, i.e. $S J=-J S$ (see [O]). The fundamental equations satisfied by $\nabla, h, \mu, \nu$ and such an $S$ are the following:

$$
\begin{array}{cc}
R(X, Y) Z=h(Y, Z) S X-h(X, Z) S Y+h(J Y, Z) S J X-h(J X, Z) S J Y & (\text { Gauss }), \\
\left(\nabla_{X} h\right)(Y, Z)+\mu(X) h(Y, Z)+\nu(X) h(J Y, Z) & (\text { Codazzi } I), \\
=\left(\nabla_{Y} h\right)(X, Z)+\mu(Y) h(X, Z)+\nu(Y) h(J X, Z) & (\text { Codazzi II }), \\
\left(\nabla_{X} S\right) Y-\mu(X) S Y+\nu(X) S J Y=\left(\nabla_{Y} S\right) X-\mu(Y) S X+\nu(Y) S J X \\
h(X, S Y)-h(Y, S X)=2 d \mu(X, Y) & (\text { Ricci } I), \\
h(S X, J Y)-h(S Y, J X)=2 d \nu(X, Y) & (\text { Ricci II }) .
\end{array}
$$


Furthermore, we shall assume that for every point $m \in M$ there exists a local section $\xi: U \rightarrow \mathbb{C}^{n+1}$ of $\mathcal{N}$ with $U \ni m$ such that $\nabla S^{\xi}=0$, where $S^{\xi}$ denotes the shape operator induced by $\xi$. If the points $m_{1}, m_{2} \in U$ can be joined by a curve lying in $U$, and $\mathcal{B}_{1}$ is a basis of $T_{m_{1}} M$, then by parallel displacement we can obtain a basis $\mathcal{B}_{2}$ of $T_{m_{2}} M$ with respect to which $S_{m_{2}}^{\xi}$ has the same matrix as $S_{m_{1}}^{\xi}$ with respect to $\mathcal{B}_{1}$. Hence rank $S_{m_{1}}^{\xi}=\operatorname{rank} S_{m_{2}}^{\xi}$, where $\operatorname{rank} S_{m}^{\xi}:=\operatorname{dim}_{\mathbb{C}} \operatorname{im} S_{m}^{\xi}$. The assumed connectedness of $M$ and the independence of $\operatorname{rank} S_{m}^{\xi}$ of $\xi$ at a fixed $m$ imply that $q:=\operatorname{rank} S$ is well defined for the whole $M$.

When studying immersions with $\nabla$-parallel shape operator we shall make use of the following remarks:

REMARK 1. If $\nabla S=0$, then for every $X, Y, Z$,

$$
R(X, Y) S Z=S(R(X, Y) Z) .
$$

Proof. This is an obvious consequence of the commutativity of $S$ and $\nabla_{W}$ for any $W$.

REMARK 2. If $\nabla S=0$ and $S J=-J S$, then $\operatorname{ker} S \subset \operatorname{ker} \mu \cap \operatorname{ker} \nu$ or $S=0$.

Proof. Let $S X=0$. By the second Codazzi equation we have $-\mu(X) S Y$ $+\nu(X) S J Y=0$ for any $Y$. If $S \neq 0$, then there exists $Y$ such that $S Y \neq 0$. Since $S Y$ and $S J Y=-J S Y$ are linearly independent over $\mathbb{R}$, it follows that $\mu(X)=0$ and $\nu(X)=0$.

Remark 3. If $\nabla S=0, S J=-J S$ and $S \neq 0$, then the section $\xi$ inducing $S$ is anti-holomorphic, i.e. $\nu(X)=\mu(J X)$ for any $X$.

Proof. We may assume that $S X \neq 0$. The assertion follows easily from the second Codazzi equation, written for $Y=J X$.

For an anti-holomorphic $\xi$ we can rewrite the first Codazzi equation as

$$
\begin{aligned}
\left(\nabla_{X} h\right)(Y, Z)+\mu(X) h(Y, Z)+\mu(J X) h(J Y, Z) & \\
= & \left(\nabla_{Y} h\right)(X, Z)+\mu(Y) h(X, Z)+\mu(J Y) h(J X, Z) .
\end{aligned}
$$

3. Theorem. We can now formulate our main result.

Theorem. Let $M$ be an $n$-dimensional connected complex manifold, $n>1, f: M \rightarrow \mathbb{C}^{n+1}$ a holomorphic immersion and $\nabla$ a linear connection induced on $M$ by a transversal bundle $\mathcal{N}$. Let $f$ and $M$ satisfy the following assumptions:

(1) $t f>0$ everywhere on $M$,

(2) for every $m \in M$ there exists a neighbourhood $U$ of $m$ and a local section $\xi: U \rightarrow \mathbb{C}^{n+1}$ of $\mathcal{N}$ inducing an anti-complex and $\nabla$-parallel shape operator $S$, 
(3) $q:=\operatorname{rank} S>0$.

Under the conditions stated above,

(i) $q \leq n / 2$;

(ii) for every $m \in M$ there exists a neighbourhood $V$ of $m$, a complex chart $\widetilde{\phi}: V \rightarrow \mathbb{C}^{n} \cong \mathbb{C}^{q} \times \mathbb{C}^{n-2 q} \times \mathbb{C}^{q}$, a complex affine isomorphism $\widetilde{A}: \mathbb{C}^{n+1} \rightarrow \mathbb{C}^{n+1}$, and a holomorphic function $\widetilde{\mathcal{F}}$ such that

$$
\begin{aligned}
& \widetilde{A} \circ f \circ \widetilde{\phi}^{-1}\left(\widetilde{x}^{1}, \ldots, \widetilde{x}^{q}, \widetilde{y}^{1}, \ldots, \widetilde{y}^{n-2 q}, \widetilde{z}^{1}, \ldots, \widetilde{z}^{q}\right) \\
& =\left(\widetilde{x}^{1}, \ldots, \widetilde{x}^{q}, \widetilde{y}^{1}, \ldots, \widetilde{y}^{n-2 q}, \widetilde{z}^{1}, \ldots, \widetilde{z}^{q}, \widetilde{\mathcal{F}}\left(\widetilde{y}^{1}, \ldots, \widetilde{y}^{n-2 q}, \widetilde{z}^{1}, \ldots, \widetilde{z}^{q}\right)\right)
\end{aligned}
$$

(iii) if $q>1$, then the local section $\overrightarrow{\widetilde{A}} \circ \xi: V \rightarrow \mathbb{C}^{n+1}$ of $\overrightarrow{\widetilde{A}} \mathcal{N}$ (where $\overrightarrow{\widetilde{A}}$ denotes the linear part of $\widetilde{A})$ inducing the $\nabla$-parallel shape operator is described in this chart by the following formula:

$$
\overrightarrow{\widetilde{A}} \circ \xi \circ \widetilde{\phi}^{-1}\left(\widetilde{x}^{1}, \ldots, \widetilde{x}^{q}, \widetilde{y}^{1}, \ldots, \widetilde{y}^{n-2 q}, \widetilde{z}^{1}, \ldots, \widetilde{z}^{q}\right)=(\overline{\widetilde{z}^{1}}, \ldots, \overline{\widetilde{z}^{q}}, \underbrace{0, \ldots, 0}_{n-q \text { times }}, 1) ;
$$

(iv) if $q=1$, then

$$
\overrightarrow{\widetilde{A}} \circ \xi \circ \widetilde{\phi}^{-1}\left(\widetilde{x}, \widetilde{y}^{1}, \ldots, \widetilde{y}^{n-2}, \widetilde{z}\right)=(\overline{\widetilde{\mathcal{G}}(\widetilde{z})}, \underbrace{0, \ldots, 0}_{n-1 \text { times }}, \overline{e^{\overline{\mathcal{M}}(\widetilde{z})}})
$$

where $\widetilde{\mathcal{G}}$ and $\widetilde{\mathcal{M}}$ are holomorphic functions such that

$$
\widetilde{\mathcal{G}}^{\prime}(\widetilde{z})-\widetilde{\mathcal{M}^{\prime}}(\widetilde{z}) \widetilde{\mathcal{G}}(\widetilde{z}) \equiv 1
$$

In the real representation, setting $\widetilde{x}^{k}=x^{2 k-1}+i x^{2 k}, \widetilde{y}^{l}=y^{2 l-1}+i y^{2 l}$, $\widetilde{z}^{j}=z^{2 j-1}+i z^{2 j}$ for $j, k=1, \ldots, q ; l=1, \ldots, n-2 q ; \widetilde{\mathcal{F}}=\mathcal{F}^{1}+i \mathcal{F}^{2}$, $\widetilde{\mathcal{G}}=\mathcal{G}^{1}+i \mathcal{G}^{2}, \widetilde{\mathcal{M}}=\mathcal{M}^{1}+i \mathcal{M}^{2}$, we have

$$
\begin{aligned}
& A \circ f \circ \phi^{-1}\left(x^{1}, \ldots, x^{2 q}, y^{1}, \ldots, y^{2 n-4 q}, z^{1}, \ldots, z^{2 q}\right) \\
& \quad=\left(x^{1}, \ldots, x^{2 q}, y^{1}, \ldots, y^{2 n-4 q}, z^{1}, \ldots, z^{2 q}, \mathcal{F}^{1}(y, z), \mathcal{F}^{2}(y, z)\right), \\
& \vec{A} \circ \xi \circ \phi^{-1}\left(x^{1}, \ldots, x^{2 q}, y^{1}, \ldots, y^{2 n-4 q}, z^{1}, \ldots, z^{2 q}\right) \\
& \quad=(z^{1},-z^{2}, \ldots, z^{2 q-1},-z^{2 q}, \underbrace{0, \ldots, 0}_{2 n-2 q \text { times }}, 1,0)
\end{aligned}
$$

if $q>1$, and

$$
\begin{aligned}
& \vec{A} \circ \xi \circ \phi^{-1}\left(x^{1}, x^{2}, y^{1}, \ldots, y^{2 n-4}, z^{1}, z^{2}\right) \\
& \quad(\mathcal{G}^{1}(z),-\mathcal{G}^{2}(z), \underbrace{0, \ldots, 0}_{2 n-2 \text { times }}, e^{\mathcal{M}^{1}(z)} \cos \mathcal{M}^{2}(z),-e^{\mathcal{M}^{1}(z)} \sin \mathcal{M}^{2}(z))
\end{aligned}
$$

if $q=1$. 
REMARK 4. An easy computation shows that the converse is also true:

(a) For any holomorphic function $\widetilde{\mathcal{F}}$ of $n-q$ variables, where $q \leq n / 2$, the shape operator $S$ induced on the hypersurface

$$
\begin{aligned}
f: \mathbb{C}^{n} \supset U & \ni\left(\widetilde{x}^{1}, \ldots, \widetilde{x}^{q}, \widetilde{y}^{1}, \ldots, \widetilde{y}^{n-2 q}, \widetilde{z}^{1}, \ldots, \widetilde{z}^{q}\right) \\
& \mapsto\left(\widetilde{x}^{1}, \ldots, \widetilde{x}^{q}, \widetilde{y}^{1}, \ldots, \widetilde{y}^{n-2 q}, \widetilde{z}^{1}, \ldots, \widetilde{z}^{q}, \widetilde{\mathcal{F}}(\widetilde{y}, \widetilde{z})\right) \in \mathbb{C}^{n+1}
\end{aligned}
$$

endowed with the transversal field

$$
\xi\left(\widetilde{x}^{1}, \ldots, \widetilde{x}^{q}, \widetilde{y}^{1}, \ldots, \widetilde{y}^{n-2 q}, \widetilde{z}^{1}, \ldots, \widetilde{z}^{q}\right)=(\overline{\widetilde{z}^{1}}, \ldots, \overline{\widetilde{z}^{q}}, \underbrace{0, \ldots, 0}_{n-q \text { times }}, 1)
$$

is parallel with respect to the induced connection and $\operatorname{rank} S=q$.

(b) For any holomorphic function $\widetilde{\mathcal{F}}$ of $n-1$ variables and for any holomorphic functions $\widetilde{\mathcal{G}}$ and $\widetilde{\mathcal{M}}$ of one variable satisfying the equation

$$
\widetilde{\mathcal{G}}^{\prime}(\widetilde{z})-\widetilde{\mathcal{M}^{\prime}}(\widetilde{z}) \widetilde{\mathcal{G}}(\widetilde{z}) \equiv 1
$$

the shape operator $S$ induced on the hypersurface

$$
f: \mathbb{C}^{n} \supset U \ni\left(\widetilde{x}, \widetilde{y}^{1}, \ldots, \widetilde{y}^{n-2}, \widetilde{z}\right) \mapsto\left(\widetilde{x}, \widetilde{y}^{1}, \ldots, \widetilde{y}^{n-2}, \widetilde{z}, \widetilde{\mathcal{F}}(\widetilde{y}, \widetilde{z})\right) \in \mathbb{C}^{n+1}
$$

endowed with the transversal field

$$
\xi\left(\widetilde{x}, \widetilde{y}^{1}, \ldots, \widetilde{y}^{n-2}, \widetilde{z}\right)=(\overline{\widetilde{\mathcal{G}}(\widetilde{z})}, \underbrace{0, \ldots, 0}_{n-1 \text { times }}, \overline{e^{\widetilde{\mathcal{M}}(\widetilde{z}}})
$$

is parallel with respect to the induced connection and has $\operatorname{rank} S=1$.

Proof of the Theorem. We begin by proving two lemmas in which we establish some inclusions between $\operatorname{im} S$, $\operatorname{ker} S$ and $\operatorname{ker} h$.

Lemma 1. If $t f>0, S J=-J S$, and $\nabla S=0$, then the following conditions are equivalent:

(1) $d \mu=0$ and $d \nu=0$,

(2) $\operatorname{im} S \subset \operatorname{ker} h$,

(3) $\operatorname{im} S \subset \operatorname{ker} S$.

Proof. (1) $\Leftrightarrow(2)$. Suppose that $d \mu=0$ and $d \nu=0$. Let $m^{\prime} \in M$ and $X, Y \in T_{m^{\prime}} M$. Applying the Ricci equations we have

$$
\begin{aligned}
0 & =2 d \mu(X, Y)+2 d \nu(J X, Y) \\
& =h(X, S Y)-h(Y, S X)-h\left(J^{2} X, S Y\right)+h(J Y, S J X) \\
& =h(X, S Y)-h(Y, S X)+h(X, S Y)+h(J Y,-J S X) \\
& =h(X, S Y)-h(Y, S X)+h(X, S Y)+h(Y, S X)=2 h(X, S Y),
\end{aligned}
$$

hence for any $X, Y \in T_{m^{\prime}} M$ we have $h(X, S Y)=0$. The Ricci equations make it obvious that (2) implies (1). 
(2) $\Rightarrow(3)$. Suppose that $\operatorname{im} S \subset \operatorname{ker} h$, which yields $R(X, Y) S Z=0$ by the Gauss equation. Since $t f_{m^{\prime}}>0$, there exist $X_{0}, Y_{0} \in T_{m^{\prime}} M$ such that $h\left(X_{0}, Y_{0}\right) \neq 0$. We first show that $S^{2} X_{0}=0$. Indeed, making use of Remark 1 we have

$$
0=S\left(R\left(X_{0}, J X_{0}\right) Y_{0}\right)=2\left(h\left(J X_{0}, Y_{0}\right) S^{2} X_{0}-h\left(X_{0}, Y_{0}\right) S^{2} J X_{0}\right)
$$

with $h\left(X_{0}, Y_{0}\right) \neq 0$, which means that $S^{2} X_{0}$ and $S^{2} J X_{0}$ are linearly dependent over $\mathbb{R}$. This is possible only when $S^{2} X_{0}=S^{2} J X_{0}=0$.

Now we take an arbitrary $Z \in T_{m^{\prime}} M$. Then

$$
0=S\left(R\left(X_{0}, Z\right) Y_{0}\right)=-h\left(X_{0}, Y_{0}\right) S^{2} Z-h\left(J X_{0}, Y_{0}\right) S^{2} J Z,
$$

and by a similar argument $S^{2} Z=0$.

(3) $\Rightarrow(2)$. If $S^{2}=0$, then the right-hand side of the equality $R(X, Y) S Z$ $=S(R(X, Y) Z)$ vanishes for every $X, Y, Z$. For $S=0$, (2) holds, therefore we can assume that $S \neq 0$. Take $X_{0}$ such that $S X_{0} \neq 0$. Since

$$
0=R\left(X_{0}, J X_{0}\right) S Z=2\left(h\left(J X_{0}, S Z\right) S X_{0}-h\left(X_{0}, S Z\right) S J X_{0}\right)
$$

and $S X_{0}, S J X_{0}$ are linearly independent over $\mathbb{R}$, we have

$$
h\left(X_{0}, S Z\right)=h\left(J X_{0}, S Z\right)=0 \quad \text { for any } Z \text {. }
$$

Now we can write for any $Y, Z$,

$$
0=R\left(X_{0}, Y\right) S Z=h(Y, S Z) S X_{0}+h(J Y, S Z) S J X_{0} .
$$

Hence $h(Y, S Z)=0$ for any $Y, Z$.

LEMMA 2. Under the assumptions of Lemma 1, the equivalent conditions (1), (2) and (3) are satisfied.

Proof. If $\nabla S=0$, then $\operatorname{rank} S$ is constant on the domain of $S$. We have to consider three cases.

Case 1: $\operatorname{rank} S=0$. Then, of course, (3) holds.

CASE 2: $\operatorname{rank} S=1$. Suppose, contrary to our claim, that $S^{2} \neq 0$. Let $m^{\prime} \in M$. We fix $X_{0} \in T_{m^{\prime}} M$ such that $S^{2} X_{0} \neq 0$. We shall obtain a contradiction with the assumption $t f>0$.

STEP 1. $\operatorname{ker} S \subset \operatorname{ker} h$.

Let $Z \in \operatorname{ker} S$. Then

$$
\begin{aligned}
0 & =R\left(X_{0}, J X_{0}\right) S Z=S\left(R\left(X_{0}, J X_{0}\right) Z\right) \\
& =2\left(h\left(J X_{0}, Z\right) S^{2} X_{0}-h\left(X_{0}, Z\right) S^{2} J X_{0}\right),
\end{aligned}
$$

hence $h\left(X_{0}, Z\right)=h\left(J X_{0}, Z\right)=0$. For any $Y$ we now have

$$
0=R\left(X_{0}, Y\right) S Z=S\left(R\left(X_{0}, Y\right) Z\right)=h(Y, Z) S^{2} X_{0}+h(J Y, Z) S^{2} J X_{0},
$$

which implies $h(Y, Z)=0$ for any $Z \in \operatorname{ker} S$ and for any $Y$.

Step 2. (a) $T_{m^{\prime}} M=\operatorname{ker} S \oplus \mathbb{C} X_{0}$ and (b) $T_{m^{\prime}} M=\operatorname{ker} S \oplus \mathbb{C} S X_{0}$. 
Since $\operatorname{dim}_{\mathbb{C}} \operatorname{ker} S=n-1$, it is sufficient and easy to check that ker $S \cap$ $\mathbb{C} X_{0}=\{0\}$ and ker $S \cap \mathbb{C} S X_{0}=\{0\}$.

SteP 3. $h\left(X_{0}, S X_{0}\right)=h\left(J X_{0}, S J X_{0}\right)=0$.

If $h\left(X_{0}, X_{0}\right)=0$ and $h\left(X_{0}, J X_{0}\right)=0$ then $X_{0} \in \operatorname{ker} h$ by Steps 1 and $2(\mathrm{a})$, and so the claimed equality holds.

Assume now that $h\left(X_{0}, X_{0}\right) \neq 0$ or $h\left(X_{0}, J X_{0}\right) \neq 0$. We have

$$
\begin{aligned}
0= & R\left(X_{0}, J X_{0}\right) S X_{0}-S\left(R\left(X_{0}, J X_{0}\right) X_{0}\right. \\
= & 2 S\left(h\left(J X_{0}, S X_{0}\right) X_{0}-h\left(X_{0}, S X_{0}\right) J X_{0}\right. \\
& \left.-h\left(J X_{0}, X_{0}\right) S X_{0}+h\left(X_{0}, X_{0}\right) S J X_{0}\right),
\end{aligned}
$$

therefore $Z_{0} \in \operatorname{ker} S$, where

$$
\begin{aligned}
Z_{0}:= & h\left(J X_{0}, S X_{0}\right) X_{0}-h\left(X_{0}, S X_{0}\right) J X_{0} \\
& -h\left(J X_{0}, X_{0}\right) S X_{0}+h\left(X_{0}, X_{0}\right) S J X_{0} .
\end{aligned}
$$

According to Step 1, we have $h\left(Z_{0}, X_{0}\right)=0$ and $h\left(Z_{0}, J X_{0}\right)=0$, hence

$$
\begin{aligned}
& h\left(J X_{0}, S X_{0}\right) h\left(X_{0}, X_{0}\right)-h\left(X_{0}, S X_{0}\right) h\left(J X_{0}, X_{0}\right) \\
& -h\left(J X_{0}, X_{0}\right) h\left(S X_{0}, X_{0}\right)+h\left(X_{0}, X_{0}\right) h\left(S J X_{0}, X_{0}\right)=0
\end{aligned}
$$

and

$$
\begin{aligned}
& h\left(J X_{0}, S X_{0}\right) h\left(X_{0}, J X_{0}\right)-h\left(X_{0}, S X_{0}\right) h\left(J X_{0}, J X_{0}\right) \\
& \quad-h\left(J X_{0}, X_{0}\right) h\left(S X_{0}, J X_{0}\right)+h\left(X_{0}, X_{0}\right) h\left(S J X_{0}, J X_{0}\right)=0 .
\end{aligned}
$$

Thus we obtain

$$
h\left(S X_{0}, X_{0}\right) h\left(J X_{0}, X_{0}\right)=0 \quad \text { and } h\left(S X_{0}, X_{0}\right) h\left(X_{0}, X_{0}\right)=0,
$$

which implies $h\left(S X_{0}, X_{0}\right)=0$, and consequently, by the anti-complexity of $S$ and the properties of $h(\cdot, \cdot), h\left(S J X_{0}, J X_{0}\right)=0$.

STEP 4. $h(Z, S W)+h(W, S Z)=0$ for any $Z, W \in T_{m^{\prime}} M$.

By Step 2(a) we have $Z=Z_{1}+\alpha X_{0}+\beta J X_{0}, W=W_{1}+\gamma X_{0}+\delta J X_{0}$ with $Z_{1}, W_{1} \in \operatorname{ker} S$ and $\alpha, \beta, \gamma, \delta \in \mathbb{R}$. An easy computation gives

$$
\begin{aligned}
& h(Z, S W)+h(W, S Z) \\
& =h\left(\alpha X_{0}+\beta J X_{0}, S\left(\gamma X_{0}+\delta J X_{0}\right)\right)+h\left(\gamma X_{0}+\delta J X_{0}, S\left(\alpha X_{0}+\beta J X_{0}\right)\right) \\
& =2 \alpha \gamma h\left(X_{0}, S X_{0}\right)+2 \beta \delta h\left(J X_{0}, S J X_{0}\right)+(\alpha \delta+\beta \gamma) h\left(X_{0},(S J+J S) X_{0}\right),
\end{aligned}
$$

which vanishes by the anti-complexity of $S$ and by Step 3 .

STEP 5. $\left(\nabla_{W} h\right)\left(X_{0}, S X_{0}\right)=\left(\nabla_{W} h\right)\left(J X_{0}, S J X_{0}\right)=0$ for any $W$.

We extend $X_{0}$ to a local vector field $X_{0}$ such that $S^{2} X_{0} \neq 0$ at any point of the domain of $X_{0}$. We have 


$$
\begin{aligned}
& \left(\nabla_{W} h\right)\left(X_{0}, S X_{0}\right) \\
& \quad=W\left(h\left(X_{0}, S X_{0}\right)\right)-h\left(\nabla_{W} X_{0}, S X_{0}\right)-h\left(X_{0}, \nabla_{W}\left(S X_{0}\right)\right) \\
& \quad=W\left(h\left(X_{0}, S X_{0}\right)\right)-\left(h\left(\nabla_{W} X_{0}, S X_{0}\right)+h\left(X_{0}, S\left(\nabla_{W} X_{0}\right)\right)\right)=0 .
\end{aligned}
$$

The same is true for $J X_{0}$ in place of $X_{0}$.

STEP 6. $\nabla h=-2 \mu \otimes h$.

If $Y \in \operatorname{ker} S_{m^{\prime}}$, then we can extend $Y$ to a local section $Y$ of ker $S$. For any $X, Z, \nabla_{X} Y \in \operatorname{ker} S$ and

$$
\begin{aligned}
\left(\nabla_{X} h\right)(Y, Z) & =X(h(Y, Z))-h\left(\nabla_{X} Y, Z\right)-h\left(Y, \nabla_{X} Z\right)=0 \\
& =-2 \mu(X) h(Y, Z) .
\end{aligned}
$$

Let $X \in \operatorname{ker} S$. Then for any $Y, Z$ we have

$$
\begin{aligned}
\left(\nabla_{X} h\right)(Y, Z) \underset{\text { Codazzi I }}{=} & -\mu(X) h(Y, Z)-\mu(J X) h(J Y, Z) \\
& +\left(\nabla_{Y} h\right)(X, Z)+\mu(Y) h(X, Z)+\mu(J Y) h(J X, Z) \\
= & 0=-2 \mu(X) h(Y, Z),
\end{aligned}
$$

since ker $S \subset \operatorname{ker} \mu \cap \operatorname{ker} \nu$ and ker $S \subset \operatorname{ker} h$.

It follows that $\left(\nabla_{X} h\right)(Y, \cdot)=-2 \mu(X) h(Y, \cdot)$ if $X \in \operatorname{ker} S$ or $Y \in \operatorname{ker} S$.

From the first Codazzi equation we obtain

$$
\begin{aligned}
& \left(\nabla_{X_{0}} h\right)\left(J X_{0}, S X_{0}\right)+\mu\left(X_{0}\right) h\left(J X_{0}, S X_{0}\right)+\mu\left(J X_{0}\right) h\left(J^{2} X_{0}, S X_{0}\right) \\
& =\left(\nabla_{J X_{0}} h\right)\left(X_{0}, S X_{0}\right)+\mu\left(J X_{0}\right) h\left(X_{0}, S X_{0}\right)+\mu\left(J^{2} X_{0}\right) h\left(J X_{0}, S X_{0}\right) .
\end{aligned}
$$

Hence

$$
\left(\nabla_{X_{0}} h\right)\left(J X_{0}, S X_{0}\right)=-2 \mu\left(X_{0}\right) h\left(J X_{0}, S X_{0}\right),
$$

by Steps 3 and 5 .

Similarly,

$$
\begin{aligned}
& \left(\nabla_{X_{0}} h\right)\left(J X_{0}, S J X_{0}\right)+\mu\left(X_{0}\right) h\left(J X_{0}, S J X_{0}\right)+\mu\left(J X_{0}\right) h\left(J^{2} X_{0}, S J X_{0}\right) \\
& \quad=\left(\nabla_{J X_{0}} h\right)\left(X_{0}, S J X_{0}\right)+\mu\left(J X_{0}\right) h\left(X_{0}, S J X_{0}\right)+\mu\left(J^{2} X_{0}\right) h\left(J X_{0}, S J X_{0}\right),
\end{aligned}
$$

which gives

$$
\left(\nabla_{J X_{0}} h\right)\left(X_{0}, S J X_{0}\right)=-2 \mu\left(J X_{0}\right) h\left(X_{0}, S J X_{0}\right) .
$$

We now have

$$
\begin{gathered}
\left(\nabla_{X_{0}} h\right)\left(X_{0}, S X_{0}\right)=0=-2 \mu\left(X_{0}\right) h\left(X_{0}, S X_{0}\right), \\
\left(\nabla_{X_{0}} h\right)\left(X_{0}, S J X_{0}\right)=-\left(\nabla_{X_{0}} h\right)\left(J X_{0}, S X_{0}\right)=2 \mu\left(X_{0}\right) h\left(J X_{0}, S X_{0}\right) \\
=-2 \mu\left(X_{0}\right) h\left(X_{0}, S J X_{0}\right)
\end{gathered}
$$

and

$$
\left(\nabla_{X_{0}} h\right)\left(X_{0}, Z\right)=-2 \mu\left(X_{0}\right) h\left(X_{0}, Z\right)
$$


for $Z \in \operatorname{ker} S$. Therefore

$$
\left(\nabla_{X_{0}} h\right)\left(X_{0}, \cdot\right)=-2 \mu\left(X_{0}\right) h\left(X_{0}, \cdot\right)
$$

by Step 2(b).

In the same manner we can see that

$$
\begin{aligned}
\left(\nabla_{X_{0}} h\right)\left(J X_{0}, \cdot\right) & =-2 \mu\left(X_{0}\right) h\left(J X_{0}, \cdot\right), \\
\left(\nabla_{J X_{0}} h\right)\left(X_{0}, \cdot\right) & =-2 \mu\left(J X_{0}\right) h\left(X_{0}, \cdot\right), \\
\left(\nabla_{J X_{0}} h\right)\left(J X_{0}, \cdot\right) & =-2 \mu\left(J X_{0}\right) h\left(J X_{0}, \cdot\right),
\end{aligned}
$$

which completes the proof of Step 6 .

As a consequence of Step 6 we obtain

Step 7. $R(X, Y) \cdot h=-4 d \mu(X, Y) h$ for any $X, Y$.

Applying the Ricci equation yields

STEP 8. $R(X, Y) \cdot h=-2(h(X, S Y)-h(Y, S X)) h$. In particular, we have $R\left(X_{0}, J X_{0}\right) \cdot h=-4 h\left(X_{0}, S J X_{0}\right) h$.

On the other hand, a direct computation gives

$$
\begin{aligned}
\left(R\left(X_{0}, J X_{0}\right)\right. & \cdot h)\left(X_{0}, X_{0}\right) \\
& =-2 h\left(R\left(X_{0}, J X_{0}\right) X_{0}, X_{0}\right) \\
& =-2 h\left(2\left(h\left(J X_{0}, X_{0}\right) S X_{0}-h\left(X_{0}, X_{0}\right) S J X_{0}\right), X_{0}\right) \\
& =-4 h\left(J X_{0}, X_{0}\right) h\left(S X_{0}, X_{0}\right)+4 h\left(X_{0}, X_{0}\right) h\left(S J X_{0}, X_{0}\right) \\
& =4 h\left(X_{0}, X_{0}\right) h\left(X_{0}, S J X_{0}\right),
\end{aligned}
$$

and

$$
\begin{aligned}
&\left(R\left(X_{0}, J X_{0}\right) \cdot h\right)\left(X_{0}, J X_{0}\right) \\
&=-h\left(R\left(X_{0}, J X_{0}\right) X_{0}, J X_{0}\right)-h\left(X_{0}, R\left(X_{0}, J X_{0}\right) J X_{0}\right) \\
&=-h\left(2\left(h\left(J X_{0}, X_{0}\right) S X_{0}-h\left(X_{0}, X_{0}\right) S J X_{0}\right), J X_{0}\right) \\
&-h\left(X_{0}, 2\left(h\left(J X_{0}, J X_{0}\right) S X_{0}-h\left(X_{0}, J X_{0}\right) S J X_{0}\right)\right) \\
&=-2 h\left(J X_{0}, X_{0}\right) h\left(S X_{0}, J X_{0}\right)+2 h\left(X_{0}, X_{0}\right) h\left(S J X_{0}, J X_{0}\right) \\
&-2 h\left(J X_{0}, J X_{0}\right) h\left(X_{0}, S X_{0}\right)+2 h\left(X_{0}, J X_{0}\right) h\left(X_{0}, S J X_{0}\right) \\
&= 4 h\left(J X_{0}, X_{0}\right) h\left(X_{0}, S J X_{0}\right) .
\end{aligned}
$$

A comparison with Step 8 gives

$$
h\left(X_{0}, S J X_{0}\right)=0 \quad \text { or } \quad h\left(X_{0}, X_{0}\right)=h\left(X_{0}, J X_{0}\right)=0,
$$

which together with Steps 1-3 leads to a contradiction with the assumption $h_{m^{\prime}} \neq 0$. 
CASE 3: $\operatorname{rank} S>1$. If $S X=0$, then, by Remark $2, \mu(X)=\nu(X)=0$. Let $S X \neq 0$. Then there exists $Y$ such that $S X$ and $S Y$ are linearly independent over $\mathbb{C}$. From Codazzi II we have

$$
(\mu(X)+i \nu(X)) S Y-(\mu(Y)+i \nu(Y)) S X=0,
$$

which implies $\mu(X)=\nu(X)=0$. Therefore (1) of Lemma 1 holds.

We now return to the proof of the theorem.

Fix $m \in M$. Let $\xi: U \rightarrow \mathbb{C}^{n+1}$ be defined on a connected neighbourhood $U$ of $m$ and have the property described in assumption (2) of the Theorem.

Lemma 3. There exists a q-dimensional complex subspace $\mathcal{W}$ of $\mathbb{C}^{n+1}$ such that $f_{*} \operatorname{im} S_{m^{\prime}}=\mathcal{W}$ for every $m^{\prime} \in U$.

Proof. It is sufficient to show that $f_{*} \operatorname{im} S_{m}=f_{*} \operatorname{im} S_{m^{\prime}}$. Let $\gamma:[0,1] \rightarrow$ $U$ be a $\mathcal{C}^{1}$ curve joining $m$ and $m^{\prime} ; \gamma(0)=m, \gamma(1)=m^{\prime}$. We choose $X_{1 m}, \ldots, X_{q m} \in T_{m} M$ such that $S X_{1 m}, \ldots, S X_{q m}$ form a basis over $\mathbb{C}$ of $f_{*} \operatorname{im} S_{m}$. Let $\widetilde{X}_{1}, \ldots, \widetilde{X}_{q}$ be the vector fields defined along the curve $\gamma$, parallel with respect to $\nabla, \widetilde{X}_{i}(0)=X_{i m}$ for $i \in\{1, \ldots, q\}$. It is easy to check that the map

$$
[0,1] \ni t \mapsto f_{*} S_{\gamma(t)} \widetilde{X}_{i}(t) \in \mathbb{C}^{n+1}
$$

is constant. Indeed,

$$
\begin{aligned}
\frac{d}{d t}\left(t \mapsto f_{*} S_{\gamma(t)} \widetilde{X}_{i}(t)\right)= & D_{\dot{\gamma}(t)} f_{*} S \widetilde{X}_{i} \\
= & f_{*} \nabla_{\dot{\gamma}(t)} S \widetilde{X}_{i}+h\left(\dot{\gamma}(t), S \widetilde{X}_{i}(t)\right) \xi_{\gamma(t)} \\
& -h\left(J \dot{\gamma}(t), S \widetilde{X}_{i}(t)\right) J \xi_{\gamma(t)} .
\end{aligned}
$$

The last two terms vanish because $\operatorname{im} S \subset \operatorname{ker} h$, and $\nabla_{\dot{\gamma}(t)} S \widetilde{X}_{i}=S \nabla_{\dot{\gamma}(t)} \widetilde{X}_{i}$ $=S 0=0$. It follows that

$$
\operatorname{span}_{\mathbb{C}}\left\{f_{*} S_{m} \widetilde{X}_{i}(0): i=1, \ldots, q\right\}=\operatorname{span}_{\mathbb{C}}\left\{f_{*} S_{m^{\prime}} \widetilde{X}_{i}(1): i=1, \ldots, q\right\},
$$

that is, $f_{*} \operatorname{im} S_{m}=f_{*} \operatorname{im} S_{m^{\prime}}=: \mathcal{W}$.

Let $\widetilde{A}_{1}: \mathbb{C}^{n+1} \rightarrow \mathbb{C}^{n+1}$ be a linear isomorphism such that

$$
\widetilde{A}_{1} \mathcal{W}=\operatorname{span}_{\mathbb{C}}\left\{\widetilde{e}_{1}, \ldots, \widetilde{e}_{q}\right\} .
$$

Here and subsequently $\widetilde{e}_{1}, \ldots, \widetilde{e}_{n+1}$ denotes the standard basis of $\mathbb{C}^{n+1}$, whereas $e_{1}, \ldots, e_{2 n+2}$ is the standard basis of $\mathbb{R}^{2 n+2}$.

Lemma 4. There exists an $i_{0} \in\{q+1, \ldots, n+1\}$ such that $\widetilde{e}_{i_{0}} \notin$ $\left(\widetilde{A}_{1} \circ f\right)_{*} T_{m} M$ and the $i_{0}$ th coordinate of $\widetilde{A}_{1} \xi_{m}$ does not vanish.

Proof. Suppose that the assertion is false. Then $\widetilde{e}_{j} \in\left(\widetilde{A}_{1} \circ f\right)_{*} T_{m} M$ for every $j \in\{q+1, \ldots, n+1\}$ such that the $j$ th coordinate of $\widetilde{A}_{1} \xi$ does not 
vanish at $m$. Then obviously $\widetilde{A}_{1} \xi_{m} \in\left(\widetilde{A}_{1} \circ f\right)_{*} T_{m} M$, which contradicts the transversality of $\xi$.

Let $\widetilde{A}_{2}^{0}$ be the linear isomorphism of $\mathbb{C}^{n+1}$ defined by

$$
\widetilde{A}_{2}^{0} \widetilde{e}_{k}:= \begin{cases}\widetilde{e}_{k} & \text { if } k \notin\left\{i_{0}, n+1\right\} \\ \widetilde{e}_{n+1} & \text { if } k=i_{0}, \\ \widetilde{e}_{i_{0}} & \text { if } k=n+1 .\end{cases}
$$

Let $\widetilde{A}_{2}:=\widetilde{A}_{2}^{0} \circ \widetilde{A}_{1}$. Now $\widetilde{e}_{n+1}$ is transversal to $\left(\widetilde{A}_{2} \circ f\right)_{*} T_{m} M$ and $\widetilde{A}_{2} \xi$ has the non-vanishing $(n+1)$ th coordinate at $m$. Moreover, the isomorphism $\widetilde{A}_{2}^{0}$ does not change the subspace $\left(\widetilde{A}_{1} \circ f\right)_{*} \operatorname{im} S=\operatorname{span}_{\mathbb{C}}\left\{\widetilde{e}_{1}, \ldots, \widetilde{e}_{q}\right\}$.

We denote by $\pi$ the projection $\pi: \mathbb{C}^{n+1} \ni\left(\zeta^{1}, \ldots, \zeta^{n+1}\right) \mapsto\left(\zeta^{1}, \ldots, \zeta^{n}\right)$ $\in \mathbb{C}^{n}$. It is easy to check that

$$
d_{m}\left(\pi \circ \widetilde{A}_{2} \circ f\right): T_{m} M \rightarrow \mathbb{C}^{n}
$$

is a monomorphism. Indeed, if $d_{m}\left(\pi \circ \widetilde{A}_{2} \circ f\right) . V=0$, then $\left(\widetilde{A}_{2} \circ f\right)_{*} V \in \mathbb{C} \widetilde{e}_{n+1}$. $\operatorname{But}\left(\widetilde{A}_{2} \circ f\right)_{*} T_{m} M \cap \mathbb{C} \widetilde{e}_{n+1}=\{0\}$ and $\left(\widetilde{A}_{2} \circ f\right)_{*}$ is a monomorphism; therefore $V=0$.

We can now take $\widetilde{\phi}_{1}:=\pi \circ \widetilde{A}_{2} \circ f$ as a complex chart on some neighbourhood $U_{1} \subset U$ of $m$. In this chart

$$
\widetilde{A}_{2} \circ f \circ \widetilde{\phi}_{1}^{-1}\left(\zeta^{1}, \ldots, \zeta^{n}\right)=\left(\zeta^{1}, \ldots, \zeta^{n}, \widetilde{\varphi}(\zeta)\right),
$$

with a holomorphic function $\widetilde{\varphi}$.

In the real representation, identifying $\mathbb{C}^{k}$ with $\mathbb{R}^{2 k}$,

$$
\iota_{k}: \mathbb{R}^{2 k} \ni\left(w^{1}, \ldots, w^{2 k}\right) \mapsto\left(w^{1}+i w^{2}, \ldots, w^{2 k-1}+i w^{2 k}\right) \in \mathbb{C}^{k},
$$

we can write

$$
A_{2} \circ f \circ \phi_{1}^{-1}\left(w^{1}, \ldots, w^{2 n}\right)=\left(w^{1}, \ldots, w^{2 n}, \varphi^{1}(w), \varphi^{2}(w)\right) .
$$

Here $A_{2}:=\iota_{n+1}{ }^{-1} \circ \widetilde{A}_{2}$ and $\phi_{1}:=\iota_{n}{ }^{-1} \circ \widetilde{\phi}_{1}$.

LEMma 5. (a) $\partial \varphi^{k} / \partial w^{s}=0$ for $k=1,2$ and $s=1, \ldots, 2 q$.

(b) $\operatorname{im} S=\operatorname{span}_{\mathbb{R}}\left\{\partial / \partial w^{s}: s=1, \ldots, 2 q\right\}$.

Proof. At any point $m^{\prime} \in U_{1}$ we have

$$
\left(A_{2} \circ f\right)_{*}\left(\frac{\partial}{\partial w^{s}}\right)=e_{s}+\frac{\partial \varphi^{1}}{\partial w^{s}} e_{2 n+1}+\frac{\partial \varphi^{2}}{\partial w^{s}} e_{2 n+2} .
$$

If $s \in\{1, \ldots, 2 q\}$, then $e_{s}=\left(A_{2} \circ f\right)_{*} S W_{s}$ with some $W_{s} \in T_{m^{\prime}} M$, because $e_{s} \in \operatorname{span}_{\mathbb{R}}\left\{e_{1}, \ldots, e_{2 q}\right\}=\left(A_{2} \circ f\right)_{*} \operatorname{im} S_{m^{\prime}}$. Therefore we have

$$
\left(A_{2} \circ f\right)_{*}\left(\frac{\partial}{\partial w^{s}}-S W_{s}\right)=\frac{\partial \varphi^{1}}{\partial w^{s}} e_{2 n+1}+\frac{\partial \varphi^{2}}{\partial w^{s}} e_{2 n+2} .
$$

From the transversality of $e_{2 n+1}$ and $e_{2 n+2}$ to $\left(A_{2} \circ f\right)_{*} T M$ and from the injectivity of $\left(A_{2} \circ f\right)_{*}$ it follows that (a) holds, and $\partial / \partial w^{s}-S W_{s}=0$ for 
$s=1, \ldots, 2 q$. Hence $\operatorname{span}_{\mathbb{R}}\left\{\partial / \partial w^{s}: s=1, \ldots, 2 q\right\} \subset \operatorname{im} S$, which implies (b), because the dimensions are equal.

Lemma 6. The transversal field $A_{2} \xi$ does not depend on $w^{1}, \ldots, w^{2 q}$.

Proof. We use the Weingarten formula

$$
D_{\partial / \partial w^{s}} A_{2} \xi=-\left(A_{2} \circ f\right)_{*} S \frac{\partial}{\partial w^{s}}+\mu\left(\frac{\partial}{\partial w^{s}}\right) A_{2} \xi+\nu\left(\frac{\partial}{\partial w^{s}}\right) J A_{2} \xi .
$$

According to Lemmas 5(b), 2 and Remark 2, for $s=1, \ldots, 2 q$,

$$
\frac{\partial}{\partial w^{s}} \in \operatorname{im} S \subset \operatorname{ker} S \subset \operatorname{ker} \mu \cap \operatorname{ker} \nu,
$$

hence $D_{\partial / \partial w^{s}} A_{2} \xi=0$.

We now introduce the functions $\Xi^{1}, \ldots, \Xi^{2 n+2}$ by

$$
\left(A_{2} \xi \circ \phi_{1}^{-1}\right)(w)=\sum_{k=1}^{n+1}\left[\Xi^{2 k-1}(w) e_{2 k-1}-\Xi^{2 k}(w) e_{2 k}\right] .
$$

LEMMA 7.

$$
\operatorname{rank}_{\mathbb{R}}\left[\frac{\partial \Xi^{k}}{\partial w^{j}}(w)\right]_{k=1, \ldots, 2 n+2 ; j=2 q+1, \ldots, 2 n}=2 q
$$

for $w \in \phi_{1}\left(U_{1}\right)$.

Proof. We have

$$
\begin{aligned}
\operatorname{rank}_{\mathbb{R}}\left[\frac{\partial \Xi^{k}}{\partial w^{j}}(w)\right]_{k=1, \ldots, 2 n+2 ; j=2 q+1, \ldots, 2 n} \\
=\operatorname{rank}_{\mathbb{R}}\left[(-1)^{k-1} \frac{\partial \Xi^{k}}{\partial w^{j}}(w)\right]_{k=1, \ldots, 2 n+2 ; j=2 q+1, \ldots, 2 n} \\
=\operatorname{dim}_{\mathbb{R}} \operatorname{span}\left\{D_{\partial / \partial w^{j}} A_{2} \xi: j=2 q+1, \ldots, 2 n\right\} \\
=\operatorname{dim}_{\mathbb{R}} \operatorname{span}\left\{D_{\partial / \partial w^{j}} A_{2} \xi: j=1, \ldots, 2 n\right\} \\
=\operatorname{dim}_{\mathbb{R}} \operatorname{im} S_{\phi_{1}^{-1}(w)}
\end{aligned}
$$

The last equality is due to the isomorphism of $\operatorname{im} S$ and $\operatorname{im}\left\{X \mapsto D_{X} A_{2} \xi\right\}$.

A consequence of Lemma 7 is

Corollary. $n \geq 2 q$.

LEMMA 8. $\widetilde{\Xi}^{k}:=\Xi^{2 k-1}+i \Xi^{2 k}$ is a holomorphic function for $k=$ $1, \ldots, n+1$. 
Proof. It is sufficient to show that $\Xi^{2 k-1}$ and $\Xi^{2 k}$ satisfy the CauchyRiemann equations. From Remark 3 it follows that

$$
D_{J X}\left(A_{2} \xi\right)=-J D_{X}\left(A_{2} \xi\right)
$$

for any $X \in T_{m^{\prime}} M$. Let $X=\partial / \partial w^{2 s-1}$. Then $J X=\partial / \partial w^{2 s}$ and

$$
\begin{aligned}
D_{J X}\left(A_{2} \xi\right) & =\sum_{l=1}^{n+1}\left[\frac{\partial \Xi^{2 l-1}}{\partial w^{2 s}} e_{2 l-1}-\frac{\partial \Xi^{2 l}}{\partial w^{2 s}} e_{2 l}\right], \\
-J D_{X}\left(A_{2} \xi\right) & =-J \sum_{l=1}^{n+1}\left[\frac{\partial \Xi^{2 l-1}}{\partial w^{2 s-1}} e_{2 l-1}-\frac{\partial \Xi^{2 l}}{\partial w^{2 s-1}} e_{2 l}\right] \\
& =\sum_{l=1}^{n+1}\left[-\frac{\partial \Xi^{2 l-1}}{\partial w^{2 s-1}} e_{2 l}-\frac{\partial \Xi^{2 l}}{\partial w^{2 s-1}} e_{2 l-1}\right] .
\end{aligned}
$$

Therefore

$$
\frac{\partial \Xi^{2 l-1}}{\partial w^{2 s}}=-\frac{\partial \Xi^{2 l}}{\partial w^{2 s-1}} \quad \text { and } \quad \frac{\partial \Xi^{2 l-1}}{\partial w^{2 s-1}}=\frac{\partial \Xi^{2 l}}{\partial w^{2 s}} .
$$

Now $\widetilde{\Xi}^{k}, k=1, \ldots, n+1$, are holomorphic functions of the complex variables $\zeta^{s}=w^{2 s-1}+i w^{2 s}, s=q+1, \ldots, n$. By Lemma 7 , we have

$$
\operatorname{rank}_{\mathbb{C}}\left[\frac{\partial \widetilde{\Xi}^{k}}{\partial \zeta^{l}}\right]_{k=1, \ldots, n+1 ; l=q+1, \ldots, n}=q .
$$

Lemma 9. Let $r \leq N \leq M$. Let $\mathcal{U}$ be an open set in $\mathbb{K}^{N}$, where $\mathbb{K}=\mathbb{R}$ or $\mathbb{C}$. Let $F: \mathcal{U} \rightarrow \mathbb{K}^{M}$ be a $\mathcal{C}^{1}$ mapping such that rank $F^{\prime}(x)=r$ for every $x \in \mathcal{U}$. Let $x_{0} \in \mathcal{U}$ and let $i_{1}<\ldots<i_{r}$ and $j_{1}<\ldots<j_{r}$ be chosen so that

$$
\operatorname{det}\left[\frac{\partial F^{i_{k}}}{\partial x^{j_{l}}}\left(x_{0}\right)\right]_{k=1, \ldots, r ; l=1, \ldots, r} \neq 0 .
$$

Then there exist a neighbourhood $\mathcal{U}^{\prime} \subset \mathcal{U}$ of $x_{0}$ and a diffeomorphism $\Phi:$ $\mathcal{U}^{\prime} \rightarrow \Phi\left(\mathcal{U}^{\prime}\right) \subset \mathbb{K}^{N}$ such that

$$
\left(F \circ \Phi^{-1}\right)^{i_{k}}\left(y^{1}, \ldots, y^{N}\right) \equiv y^{j_{k}} \quad \text { for } k=1, \ldots, r
$$

and

$$
\frac{\partial\left(F \circ \Phi^{-1}\right)^{k}}{\partial y^{l}} \equiv 0 \quad \text { if } l \notin\left\{j_{1}, \ldots, j_{r}\right\}, k \in\{1, \ldots, M\} .
$$

Proof. We define

$$
\widehat{\Phi}\left(x^{1}, \ldots, x^{N}\right)^{k}= \begin{cases}x^{k} & \text { if } k \notin\left\{j_{1}, \ldots, j_{r}\right\}, \\ F^{i_{s}}\left(x^{1}, \ldots, x^{N}\right) & \text { if } k=j_{s} .\end{cases}
$$

Since

$$
\operatorname{det}\left[\frac{\partial \widehat{\Phi}^{k}}{\partial x^{l}}\left(x_{0}\right)\right]_{k=1, \ldots, N ; l=1, \ldots, N}=\operatorname{det}\left[\frac{\partial F^{i_{s}}}{\partial x^{j_{s}}}\left(x_{0}\right)\right]_{s=1, \ldots, r} \neq 0
$$


there exists a neighbourhood $\mathcal{U}^{\prime} \subset \mathcal{U}$ of $x_{0}$ such that $\Phi:=\left.\widehat{\Phi}\right|_{\mathcal{U}^{\prime}}$ is a diffeomorphism.

It remains to prove that $F \circ \Phi^{-1}$ depends only on the variables $y^{j_{1}}, \ldots, y^{j_{r}}$.

Let $y \in \Phi\left(\mathcal{U}^{\prime}\right)$. Then $\left(F \circ \Phi^{-1}\right)^{\prime}(y)=F^{\prime}\left(\Phi^{-1}(y)\right) \circ\left(\Phi^{-1}\right)^{\prime}(y)$ and $\operatorname{rank}\left(F \circ \Phi^{-1}\right)^{\prime}(y)=\operatorname{rank} F^{\prime}\left(\Phi^{-1}(y)\right)=r$, because $\left(\Phi^{-1}\right)^{\prime}(y)$ is an isomorphism.

Suppose that for some $k_{0} \in\{1, \ldots, M\}$ and $l_{0} \in\{1, \ldots, N\} \backslash\left\{j_{1}, \ldots, j_{r}\right\}$,

$$
\frac{\partial\left(F \circ \Phi^{-1}\right)^{k_{0}}}{\partial y^{l_{0}}}(y) \neq 0 .
$$

Then $k_{0} \notin\left\{i_{1}, \ldots, i_{r}\right\}$ and

$$
\operatorname{det}\left[\frac{\partial\left(F \circ \Phi^{-1}\right)^{k}}{\partial y^{l}}(y)\right]_{k \in\left\{i_{1}, \ldots, i_{r}, k_{0}\right\} ; l \in\left\{j_{1}, \ldots, j_{r}, l_{0}\right\}} \neq 0,
$$

which contradicts the rank assumption.

We now restrict our attention to the case $q>1$.

LEMma 10. If $q>1$, then

(a) $\operatorname{rank}_{\mathbb{C}}\left[\partial \widetilde{\Xi}^{k} / \partial \zeta^{l}\right]_{k=1, \ldots, q ; l=q+1, \ldots, n}=q$,

(b) $\widetilde{\Xi}^{k}(\cdot)=b^{k}=$ const for $k=q+1, \ldots, n+1$,

(c) $b^{n+1} \neq 0$.

Proof. Since, for $q>1, \mu=0$ and $\nu=0$ (see proof of Lemma 2, Case 3), we have

$$
D_{\partial / \partial w^{l}} A_{2} \xi \in\left(A_{2} \circ f\right)_{*} \operatorname{im} S=\operatorname{span}_{\mathbb{R}}\left\{e_{1}, \ldots, e_{2 q}\right\},
$$

therefore $\partial \Xi^{k} / \partial w^{l}=0$ for $k=2 q+1, \ldots, 2 n+2$ and $l=2 q+1, \ldots, 2 n$, which implies

$$
\frac{\partial \widetilde{\Xi}^{k}}{\partial \zeta^{l}}=0 \quad \text { for } k=q+1, \ldots, n+1, l=q+1, \ldots, n,
$$

and

$$
\operatorname{rank}_{\mathbb{C}}\left[\frac{\partial \widetilde{\Xi}^{k}}{\partial \zeta^{l}}\right]_{k=1, \ldots, n+1 ; l=q+1, \ldots, n}=\operatorname{rank}_{\mathbb{C}}\left[\frac{\partial \widetilde{\Xi}^{k}}{\partial \zeta^{l}}\right]_{k=1, \ldots, q ; l=q+1, \ldots, n} .
$$

Point (c) is a consequence of Lemma 4 and the definition of $\widetilde{A}_{2}$.

We may now apply Lemma 9, taking $r=q, N=n, M=n+1, \mathcal{U}=$ $\widetilde{\phi}_{1}\left(U_{1}\right), \mathbb{K}=\mathbb{C}, F=\left(\widetilde{\Xi}^{1}, \ldots, \widetilde{\Xi}^{n+1}\right),\left(i_{1}, \ldots, i_{q}\right)=(1, \ldots, q)$ and $j_{1}<\ldots$ $<j_{q}$ from $\{q+1, \ldots, n\}$ chosen so that

$$
\operatorname{det}\left[\frac{\partial \widetilde{\Xi}^{k}}{\partial \zeta^{j_{s}}}\left(\widetilde{\phi}_{1}(m)\right)\right]_{k=1, \ldots, q ; s=1, \ldots, q} \neq 0 .
$$


In this way we obtain a new chart $\widetilde{\phi}_{2}:=\Phi \circ \widetilde{\phi}_{1}$ on the neighbourhood $U_{2}:=\widetilde{\phi}_{1}^{-1}\left(\mathcal{U}^{\prime}\right)$ of $m$. Since

$$
\left(\widetilde{\Xi}^{k} \circ \Phi^{-1}\right)\left(\eta^{1}, \ldots, \eta^{n}\right)= \begin{cases}\eta^{j_{k}} & \text { for } k=1, \ldots, q, \\ b^{k} & \text { for } k=q+1, \ldots, n+1,\end{cases}
$$

the transversal field is now described by the formula

$$
\left(\widetilde{A}_{2} \xi \circ \widetilde{\phi}_{2}^{-1}\right)\left(\eta^{1}, \ldots, \eta^{n}\right)=\sum_{k=1}^{q} \overline{\eta^{j_{k}}} \widetilde{e}_{k}+\sum_{k=q+1}^{n+1} \overline{b^{k}} \widetilde{e}_{k} .
$$

Let $\widetilde{A}_{3}^{0}$ be the linear isomorphism of $\mathbb{C}^{n+1}$ which transforms the basis $\left(\widetilde{e}_{1}, \ldots, \widetilde{e}_{n+1}\right)$ onto the basis

$$
\left(\widetilde{e}_{1}, \ldots, \widetilde{e}_{q}, \widetilde{e}_{q+1}, \ldots, \widehat{\widetilde{e}_{j_{1}}}, \ldots, \widehat{\widetilde{e}_{j_{q}}}, \ldots, \widetilde{e}_{n}, \widetilde{e}_{j_{1}}, \ldots, \widetilde{e}_{j_{q}}, \widetilde{e}_{n+1}\right),
$$

and let

$$
\widetilde{\phi}_{3}^{0}\left(\eta^{1}, \ldots, \eta^{n}\right):=\left(\eta^{1}, \ldots, \eta^{q}, \eta^{q+1}, \ldots, \widehat{\eta^{j_{1}}}, \ldots, \widehat{\eta^{j_{q}}}, \ldots, \eta^{n}, \eta^{j_{1}}, \ldots, \eta^{j_{q}}\right) .
$$

Taking $\widetilde{A}_{3}:=\widetilde{A}_{3}^{0} \circ \widetilde{A}_{2}, \widetilde{\phi}_{3}:=\widetilde{\phi}_{3}^{0} \circ \widetilde{\phi}_{2}$, we may write

$$
\begin{aligned}
\widetilde{A}_{3} \circ f \circ \widetilde{\phi}_{3}^{-1}\left(\widetilde{s}^{1}, \ldots, \widetilde{s}^{q}, \widetilde{t}^{1}, \ldots, \widetilde{t}^{n-2 q}, \widetilde{u}^{1}, \ldots, \widetilde{u}^{q}\right) \\
\quad=\left(\widetilde{s}^{1}, \ldots, \widetilde{s}^{q}, \widetilde{t}^{1}, \ldots, \widetilde{t}^{n-2 q}, \widetilde{\chi}^{1}(\widetilde{t}, \widetilde{u}), \ldots, \widetilde{\chi^{q}}(\widetilde{t}, \widetilde{u}), \widetilde{\varrho}(\widetilde{t}, \widetilde{u})\right)
\end{aligned}
$$

and

$$
\widetilde{A}_{3} \xi \circ \widetilde{\phi}_{3}^{-1}\left(\widetilde{s}^{1}, \ldots, \widetilde{s}^{q}, \widetilde{t}^{1}, \ldots, \widetilde{t}^{n-2 q}, \widetilde{u}^{1}, \ldots, \widetilde{u}^{q}\right)=\sum_{k=1}^{q} \overline{\widetilde{u}^{k}} \widetilde{e}_{k}+\sum_{k=q+1}^{n+1} \overline{a^{k}} \widetilde{e}_{k} .
$$

Applying now the isomorphism $\widetilde{A}_{4}^{0}$, where

$$
\widetilde{A}_{4}^{0} \widetilde{e}_{k}:= \begin{cases}\widetilde{e}_{k} & \text { for } k=1, \ldots, n, \\ \left(1 / a^{n+1}\right)\left(-\sum_{k=q+1}^{n} \overline{a^{k}} \widetilde{e}_{k}+\widetilde{e}_{n+1}\right) & \text { for } k=n+1,\end{cases}
$$

and $\widetilde{A}_{4}:=\widetilde{A}_{4}^{0} \circ \widetilde{A}_{3}$, we obtain

$$
\begin{aligned}
\widetilde{A}_{4} \circ f \circ \widetilde{\phi}_{3}^{-1}\left(\widetilde{s}^{1}, \ldots, \widetilde{s}^{q}, \widetilde{t}^{1}, \ldots, \widetilde{t}^{n-2 q}, \widetilde{u}^{1}, \ldots, \widetilde{u}^{q}\right) \\
\quad=\left(\widetilde{s}^{1}, \ldots, \widetilde{s}^{q}, \widetilde{\sigma}^{1}(\widetilde{t}, \widetilde{u}), \ldots, \widetilde{\sigma}^{n-q}(\widetilde{t}, \widetilde{u}), \widetilde{\mathcal{Q}}(\widetilde{t}, \widetilde{u})\right)
\end{aligned}
$$

and

$$
\widetilde{A}_{4} \xi \circ \widetilde{\phi}_{3}^{-1}\left(\widetilde{s}^{1}, \ldots, \widetilde{s}^{q}, \widetilde{t}^{1}, \ldots, \widetilde{t}^{n-2 q}, \widetilde{u}^{1}, \ldots, \widetilde{u}^{q}\right)=\sum_{k=1}^{q} \overline{\widetilde{u}^{k}} \widetilde{e}_{k}+\widetilde{e}_{n+1} .
$$

Here

$$
\widetilde{\sigma}^{k}(\widetilde{t}, \widetilde{u})= \begin{cases}\widetilde{t}^{k}-\frac{\overline{a^{q+k}}}{\overline{a^{n+1}}} \widetilde{\varrho}(\widetilde{t}, \widetilde{u}) & \text { for } k=1, \ldots, n-2 q, \\ \widetilde{\chi}^{k-(n-2 q)}(\widetilde{t}, \widetilde{u})-\frac{\overline{a^{q+k}}}{\overline{a^{n+1}}} \widetilde{\varrho}(\widetilde{t}, \widetilde{u}) & \text { for } k=n-2 q+1, \ldots, n-q ;\end{cases}
$$

and $\widetilde{\mathcal{Q}}=\left(1 / \overline{a^{n+1}}\right) \widetilde{\varrho}$. 
We now turn to the case $q=1$.

By Lemmas 7-9, on a connected neighbourhood $\widetilde{\widetilde{U}}_{2}$ of $m$ we may define a complex chart $\widetilde{\widetilde{\phi}}_{2}$ in which the coordinates of the transversal field are functions of one variable $\eta^{i_{0}}$ only, with $i_{0}>1$.

Next we apply the isomorphisms

$$
\widetilde{\widetilde{\phi}}_{3}^{0}\left(\eta^{1}, \ldots, \eta^{n}\right):=\left(\eta^{1}, \eta^{2}, \ldots, \widehat{\eta^{i_{0}}}, \ldots, \eta^{n}, \eta^{i_{0}}\right)
$$

and

$$
\widetilde{\widetilde{A}}_{3}^{0}\left(\theta^{1}, \ldots, \theta^{n+1}\right):=\left(\theta^{1}, \theta^{2}, \ldots, \widehat{\theta^{i_{0}}}, \ldots, \theta^{n}, \theta^{i_{0}}, \theta^{n+1}\right),
$$

to obtain

$$
\widetilde{\widetilde{A}}_{3} \circ f \circ \widetilde{\widetilde{\phi}}_{3}^{-1}\left(\widetilde{s}, \widetilde{t}^{1}, \ldots, \widetilde{t}^{n-2}, \widetilde{u}\right)=\left(\widetilde{s}, \widetilde{t}^{1}, \ldots, \widetilde{t}^{n-2}, \widetilde{\widetilde{\chi}}(\widetilde{t}, \widetilde{u}), \widetilde{\varrho}(\widetilde{t}, \widetilde{u})\right)
$$

and

$$
\widetilde{\widetilde{A}}_{3} \xi \circ{\widetilde{\phi_{3}}}_{3}^{-1}\left(\widetilde{s}, \widetilde{t}^{1}, \ldots, \widetilde{t}^{n-2}, \widetilde{u}\right)=\sum_{k=1}^{n+1} \overline{\Theta^{k}(\widetilde{u})} \widetilde{e}_{k}
$$

with $\Theta^{n+1}\left(\widetilde{\widetilde{\phi}}_{3}(m)\right) \neq 0 ; \widetilde{\widetilde{\phi}}_{3}:=\widetilde{\widetilde{\phi}}_{3}^{0} \circ \widetilde{\widetilde{\phi}}_{2}$ and $\widetilde{\widetilde{A}}_{3}:=\widetilde{\widetilde{A}}_{3}^{0} \circ \widetilde{A}_{2}$.

In the real representation $\widetilde{\widetilde{\chi}}=\chi^{1}+i \chi^{2}, \widetilde{\widetilde{\varrho}}=\varrho^{1}+i \varrho^{2}, \Theta^{k}=\vartheta^{2 k-1}+i \vartheta^{2 k}$, we have

$$
\begin{aligned}
A_{3} \circ f \circ \phi_{3}^{-1} & \left(s^{1}, s^{2}, t^{1}, \ldots, t^{2 n-4}, u^{1}, u^{2}\right) \\
= & \left(s^{1}, s^{2}, t^{1}, \ldots, t^{2 n-4}, \chi^{1}(t, u), \chi^{2}(t, u), \varrho^{1}(t, u), \varrho^{2}(t, u)\right)
\end{aligned}
$$

and

$$
A_{3} \xi \circ \phi_{3}^{-1}\left(s^{1}, s^{2}, t^{1}, \ldots, t^{2 n-4}, u^{1}, u^{2}\right)=\sum_{k=1}^{n+1}\left[\vartheta^{2 k-1}(u) e_{2 k-1}-\vartheta^{2 k}(u) e_{2 k}\right] .
$$

Let $\widehat{\pi}$ denote the projection

$$
\mathbb{C}^{n} \ni\left(\widetilde{s}, \widetilde{t}^{1}, \ldots, \widetilde{t}^{n-2}, \widetilde{u}\right) \mapsto \widetilde{u} \in \mathbb{C} .
$$

Lemma 11. There exist $c_{2}, \ldots, c_{n+1} \in \mathbb{C}, c_{n+1} \neq 0$, a neighbourhood $\widetilde{\widetilde{U}}_{3}$ of $m$ and a holomorphic function $\widetilde{\mathcal{H}}$ such that

$$
\Theta^{k}(\widetilde{u})=c_{k} e^{\widetilde{\mathcal{H}}(\widetilde{u})}
$$

for $k=1, \ldots, n+1$ and $\widetilde{u} \in \widehat{\pi}\left(\widetilde{\widetilde{\phi}}_{3}\left(\widetilde{\widetilde{U}}_{3}\right)\right)$.

Proof. We fix $j \in\{2, \ldots, n+1\}$. Since $\Theta^{j}$ is a holomorphic function, and since $\widetilde{\widetilde{U}}_{2}$ is assumed to be connected, there are two possibilities: either $\Theta^{j} \equiv 0$ on $\widehat{\pi} \circ \widetilde{\widetilde{\phi}}_{3}\left(\widetilde{\widetilde{U}}_{2}\right)$ or there exists a neighbourhood $\widetilde{W}_{j}$ of $\widetilde{u}_{0}:=\widehat{\pi}\left(\widetilde{\widetilde{\phi}}_{3}(m)\right)$ such that $\Theta^{j}(\widetilde{u}) \neq 0$ for any $\widetilde{u} \in \widetilde{W}_{j} \backslash\left\{\widetilde{u}_{0}\right\}$.

In the former case we take $c_{j}=0$. 
Suppose that $\Theta^{j} \not \equiv 0$. We can find $r>0$ such that $B\left(\widetilde{u}_{0}, r\right) \subset \widetilde{W}_{j}$. Then

$$
\widetilde{W}_{j}^{\prime}:=B\left(\widetilde{u}_{0}, r\right) \backslash\left\{\widetilde{u}: \operatorname{Re} \widetilde{u}=\operatorname{Re} \widetilde{u}_{0}, \operatorname{Im} \widetilde{u} \leq \operatorname{Im} \widetilde{u}_{0}\right\}
$$

is a simply connected domain and $\Theta^{j}(\widetilde{u}) \neq 0$ for any $\widetilde{u} \in \widetilde{W}_{j}^{\prime}$. If this is the case, there exists a holomorphic function $\lambda^{j}$ on $\widetilde{W}_{j}^{\prime}$ such that $e^{\lambda^{j}}=\left.\Theta^{j}\right|_{\widetilde{W}_{j}^{\prime}}$ and $\left(\lambda^{j}\right)^{\prime}=\Theta^{j^{\prime}} / \Theta^{j}$ on $\widetilde{W}_{j}^{\prime}$.

On the other hand, using the real representation we may write

$$
\left(\Theta^{j}\right)^{\prime}=\frac{\partial \vartheta^{2 j-1}}{\partial u^{1}}+i \frac{\partial \vartheta^{2 j}}{\partial u^{1}} .
$$

From the Weingarten formula

$$
D_{\partial / \partial u^{1}} A_{3} \xi=-\left(A_{3} \circ f\right)_{*} S \frac{\partial}{\partial u^{1}}+\mu\left(\frac{\partial}{\partial u^{1}}\right) A_{3} \xi+\nu\left(\frac{\partial}{\partial u^{1}}\right) J A_{3} \xi
$$

it follows that for any $j \in\{2, \ldots, n+1\}$,

$$
\frac{\partial \vartheta^{2 j-1}}{\partial u^{1}}=\mu\left(\frac{\partial}{\partial u^{1}}\right) \vartheta^{2 j-1}+\nu\left(\frac{\partial}{\partial u^{1}}\right) \vartheta^{2 j}
$$

and

$$
\frac{\partial \vartheta^{2 j}}{\partial u^{1}}=\mu\left(\frac{\partial}{\partial u^{1}}\right) \vartheta^{2 j}-\nu\left(\frac{\partial}{\partial u^{1}}\right) \vartheta^{2 j-1} .
$$

Hence

$$
\left(\Theta^{j}\right)^{\prime}=\left(\mu\left(\frac{\partial}{\partial u^{1}}\right)-i \nu\left(\frac{\partial}{\partial u^{1}}\right)\right)\left(\vartheta^{2 j-1}+i \vartheta^{2 j}\right) .
$$

We may also assume that $\widetilde{\widetilde{U}}_{2}$ is simply connected, and from Lemma 2 we know that the 1 -forms $\mu, \nu$ are closed; therefore there exist functions $\mathcal{K}$ and $\mathcal{L}$ on $\widetilde{\widetilde{U}}_{2}$ such that $\mu=d \mathcal{K}, \nu=-d \mathcal{L}$. The functions $\mathcal{K} \circ \phi_{3}^{-1}$ and $\mathcal{L} \circ \phi_{3}^{-1}$ do not depend on the variables $s$ and $t$, because $\partial / \partial s^{i}, \partial / \partial t^{j} \in \operatorname{ker} S \subset \operatorname{ker} \mu \cap \operatorname{ker} \nu$,

$$
\frac{\partial \mathcal{K} \circ \phi_{3}^{-1}}{\partial s^{i}}=d \mathcal{K}\left(\frac{\partial}{\partial s^{i}}\right)=\mu\left(\frac{\partial}{\partial s^{i}}\right)=0
$$

and similarly for $\mathcal{L}$ in place of $\mathcal{K}$ or $t$ in place of $s$. It follows that there exist functions $\mathcal{H}^{1}$ and $\mathcal{H}^{2}$ defined on some open subset of $\mathbb{R}^{2}$ such that $\mathcal{K} \circ \phi_{3}^{-1}=\mathcal{H}^{1} \circ \widehat{\pi}$ and $\mathcal{L} \circ \phi_{3}^{-1}=\mathcal{H}^{2} \circ \widehat{\pi}$. We now have

$$
\mu\left(\frac{\partial}{\partial u^{s}}\right)=d \mathcal{K}\left(\left(\phi_{3}^{-1}\right)_{*} e_{2 n-2+s}\right)=d \mathcal{H}^{1}\left(\widehat{\pi}_{*} e_{2 n-2+s}\right)=d \mathcal{H}^{1}\left(e_{s}\right)=\frac{\partial \mathcal{H}^{1}}{\partial u^{s}}
$$

and

$$
\begin{aligned}
\nu\left(\frac{\partial}{\partial u^{s}}\right) & =-d \mathcal{L}\left(\left(\phi_{3}^{-1}\right)_{*} e_{2 n-2+s}\right)=-d \mathcal{H}^{2}\left(\widehat{\pi}_{*} e_{2 n-2+s}\right) \\
& =-d \mathcal{H}^{2}\left(e_{s}\right)=-\frac{\partial \mathcal{H}^{2}}{\partial u^{s}}
\end{aligned}
$$


According to Remark $3, \mathcal{H}^{1}$ and $\mathcal{H}^{2}$ satisfy the Cauchy-Riemann equations, therefore $\widetilde{\mathcal{H}}:=\mathcal{H}^{1}+i \mathcal{H}^{2}$ is a holomorphic function.

Since

$$
(\widetilde{\mathcal{H}})^{\prime}=\frac{\partial \mathcal{H}^{1}}{\partial u^{1}}+i \frac{\partial \mathcal{H}^{2}}{\partial u^{1}}=\mu\left(\frac{\partial}{\partial u^{1}}\right)-i \nu\left(\frac{\partial}{\partial u^{1}}\right)
$$

we may go back to the $\left(\Theta^{j}\right)^{\prime}$ and write

$$
\left(\Theta^{j}\right)^{\prime}=\widetilde{\mathcal{H}}^{\prime} \Theta^{j}
$$

on some neighbourhood $\widehat{\pi}\left(\widetilde{\widetilde{\phi}}_{3}\left(\widetilde{\widetilde{U}}_{3}^{\prime}\right)\right)$ of $\widetilde{u}_{0}$ (including $\left.\widetilde{u}_{0}\right)$. Comparing this with $\left(\lambda^{j}\right)^{\prime}$ we obtain $\widetilde{\mathcal{H}}^{\prime}=\left(\lambda^{j}\right)^{\prime}$ on $\widetilde{W}_{j}^{\prime \prime}:=\widetilde{W}_{j}^{\prime} \cap \widehat{\pi}\left(\widetilde{\widetilde{\phi}}_{3}\left(\widetilde{\widetilde{U}}_{3}^{\prime}\right)\right)$. Hence there exists $d_{j} \in \mathbb{C}$ such that $\lambda^{j}=\widetilde{\mathcal{H}}+d_{j}$ and

$$
\Theta^{j}=e^{\widetilde{\mathcal{H}}+d_{j}}=c_{j} e^{\tilde{\mathcal{H}}}
$$

with a non-zero constant $c_{j}:=e^{d_{j}}$. We may extend this equality from $\widetilde{W}_{j}^{\prime \prime}$ to some neighbourhood $\widehat{\pi}\left(\widetilde{\widetilde{\phi}}_{3}\left(\widetilde{\widetilde{U}}_{3}\right)\right)$ of $\widetilde{u}_{0}$, because both sides are continuous and well defined in the neighbourhood of $\widetilde{u}_{0}$. Since $\Theta^{n+1} \not \equiv 0$, we have in particular $c_{n+1} \neq 0$.

Next we use the following isomorphism of $\mathbb{C}^{n+1}$ :

$$
\widetilde{\widetilde{A}}_{4}^{0} \widetilde{e}_{k}:= \begin{cases}\widetilde{e}_{k} & \text { for } k=1, \ldots, n, \\ \left(1 / \bar{c}_{n+1}\right)\left(\widetilde{e}_{n+1}-\sum_{s=2}^{n} \bar{c}_{s} \widetilde{e}_{s}\right) & \text { for } k=n+1 .\end{cases}
$$

In this way we obtain

$$
\widetilde{\widetilde{A}}_{4} \circ f \circ \widetilde{\widetilde{\phi}}_{3}^{-1}\left(\widetilde{s}, \widetilde{t}^{1}, \ldots, \widetilde{t}^{n-2}, \widetilde{u}\right)=\left(\widetilde{s}, \widetilde{\sigma}^{1}(\widetilde{t}, \widetilde{u}), \ldots, \widetilde{\widetilde{\sigma}}^{n-1}(\widetilde{t}, \widetilde{u}), \widetilde{\widetilde{Q}}(\widetilde{t}, \widetilde{u})\right)
$$

and

$$
\widetilde{\widetilde{A}}_{4} \xi \circ \widetilde{\widetilde{\phi}}_{3}^{-1}\left(\widetilde{s}, \widetilde{t}^{1}, \ldots, \widetilde{t}^{n-2}, \widetilde{u}\right)=\overline{\Theta^{1}(\widetilde{u})} \widetilde{e}_{1}+\overline{e^{\widetilde{\mathcal{H}}(\widetilde{u})}} \widetilde{e}_{n+1},
$$

with $\widetilde{\widetilde{A}}_{4}:=\widetilde{\widetilde{A}}_{4}^{0} \circ \widetilde{\widetilde{A}}_{3}, \widetilde{\widetilde{\sigma}}^{k}(\widetilde{t}, \widetilde{u}):=\widetilde{t}^{k}-\overline{\left(c_{k+1} / c_{n+1}\right)} \widetilde{\widetilde{\varrho}}(\widetilde{t}, \widetilde{u})$ for $k=1, \ldots, n-2$, $\widetilde{\widetilde{\sigma}}^{n-1}:=\widetilde{\widetilde{\chi}}(\widetilde{t}, \widetilde{u})-\overline{\left(c_{k+1} / c_{n+1}\right)} \widetilde{\widetilde{\varrho}}(\widetilde{t}, \widetilde{u})$ and $\widetilde{\widetilde{Q}}(\widetilde{t}, \widetilde{u}):=\overline{\left(1 / c_{n+1}\right)} \widetilde{\widetilde{\varrho}}(\widetilde{t}, \widetilde{u})$.

Thus for any $q>0$ it is possible to find a map $\widehat{\phi}_{3}$ and an isomorphism $\widehat{A}_{4}$ of $\mathbb{C}^{n+1}$ such that the immersion and the transversal field have the shape

$$
\begin{aligned}
\widehat{A}_{4} \circ f \circ \widehat{\phi}_{3}^{-1}\left(\widetilde{s}^{1}, \ldots, \widetilde{s}^{q}, \widetilde{t}^{1}, \ldots, \widetilde{t}^{n-2 q}, \widetilde{u}^{1}, \ldots, \widetilde{u}^{q}\right) \\
\quad=\left(\widetilde{s}^{1}, \ldots, \widetilde{s}^{q}, \widehat{\sigma}^{1}(\widetilde{t}, \widetilde{u}), \ldots, \widehat{\sigma}^{n-q}(\widetilde{t}, \widetilde{u}), \widehat{\mathcal{Q}}(\widetilde{t}, \widetilde{u})\right)
\end{aligned}
$$

and

$$
\begin{aligned}
\widehat{A}_{4} \xi \circ \widehat{\phi}_{3}^{-1}\left(\widetilde{s}^{1}, \ldots, \widetilde{s}^{q}, \widetilde{t}^{1}, \ldots, \widetilde{t}^{n-2 q}, \widetilde{u}^{1}, \ldots, \widetilde{u}^{q}\right) \\
\quad=\sum_{k=1}^{q} \overline{\widehat{\Theta}^{k}(\widetilde{u})} \widetilde{e}_{k}+\overline{\widehat{\Theta}^{n+1}(\widetilde{u})} \widetilde{e}_{n+1},
\end{aligned}
$$

where $\widehat{\sigma}^{i}, \widehat{\mathcal{Q}}, \widehat{\Theta}^{j}$ are holomorphic functions and $\widehat{\Theta}^{n+1} \neq 0$. 
Since $\widehat{A}_{4} \circ f$ is an immersion, and $\widehat{A}_{4} \circ \xi$ is transversal to $\left(\widehat{A}_{4} \circ f\right)_{*} T M$, we have

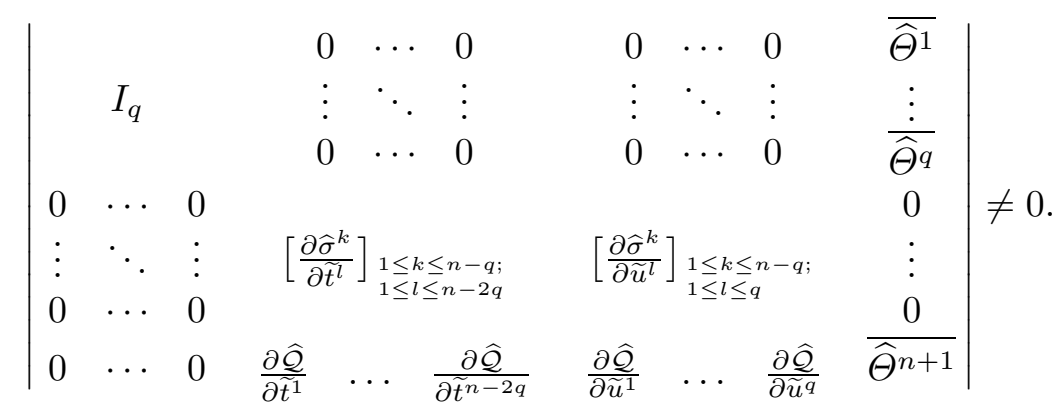

Therefore

$$
\operatorname{det}\left(\left[\frac{\partial \widehat{\sigma}^{k}}{\partial \widetilde{t}^{l}}\right]_{k=1, \ldots, n-q ; l=1, \ldots, n-2 q}\left[\frac{\partial \widehat{\sigma}^{k}}{\partial \widetilde{u}^{l}}\right]_{k=1, \ldots, n-q ; l=1, \ldots, q}\right) \neq 0
$$

and there exist $i_{1}<\ldots<i_{n-2 q}$ such that

$$
\operatorname{det}\left[\frac{\partial \widehat{\sigma}^{i_{k}}}{\partial \widetilde{t}^{l}}\right]_{k=1, \ldots, n-2 q ; l=1, \ldots, n-2 q} \neq 0 .
$$

By an appropiate isomorphism $\widehat{A}_{5}^{0}$ we may vary the order of basis vectors in $\mathbb{C}^{n+1}$, putting $\widehat{\sigma}^{i_{1}}, \ldots, \widehat{\sigma}^{i_{n-2 q}}$ at positions $q+1, \ldots, n-q$. This permutation does not affect the field $\widehat{A}_{4} \xi$, because its coordinates from the $(q+1)$ th to the $n$th vanish.

Applying now the local diffeomorphism

$$
\begin{aligned}
\widehat{\phi}_{4}^{0}\left(\widetilde{s}^{1}, \ldots, \widetilde{s}^{q}, \widetilde{t}^{1}, \ldots, \widetilde{t}^{n-2 q}, \widetilde{u}^{1}, \ldots, \widetilde{u}^{q}\right) \\
\quad:=\left(\widetilde{s}^{1}, \ldots, \widetilde{s}^{q}, \widehat{\sigma}^{i_{1}}(\widetilde{t}, \widetilde{u}), \ldots, \widehat{\sigma}^{i_{n-2 q}}(\widetilde{t}, \widetilde{u}), \widetilde{u}^{1}, \ldots, \widetilde{u}^{q}\right)
\end{aligned}
$$

gives a new chart $\widehat{\phi}_{4}:=\widehat{\phi}_{4}^{0} \circ \widehat{\phi}_{3}$ such that $\widehat{A}_{5} \circ f$ and $\widehat{A}_{5} \circ \xi$ are described by the formulas

$$
\begin{aligned}
\widehat{A}_{5} \circ f \circ \widehat{\phi}_{4}^{-1}\left(\widetilde{x}^{1}, \ldots, \widetilde{x}^{q}, \widetilde{y}^{1}, \ldots, \widetilde{y}^{n-2 q}, \widetilde{z}^{1}, \ldots, \widetilde{z}^{q}\right) \\
\quad=\left(\widetilde{x}^{1}, \ldots, \widetilde{x}^{q}, \widetilde{y}^{1}, \ldots, \widetilde{y}^{n-2 q}, \widehat{\psi}^{1}(\widetilde{y}, \widetilde{z}), \ldots, \widehat{\psi}^{q}(\widetilde{y}, \widetilde{z}), \widehat{\mathcal{F}}(\widetilde{y}, \widetilde{z})\right)
\end{aligned}
$$

and

$$
\begin{aligned}
\widehat{A}_{5} \circ \xi \circ \widehat{\phi}_{4}^{-1}\left(\widetilde{x}^{1}, \ldots, \widetilde{x}^{q}, \widetilde{y}^{1}, \ldots, \widetilde{y}^{n-2 q}, \widetilde{z}^{1}, \ldots, \widetilde{z}^{q}\right) \\
=\sum_{k=1}^{q} \overline{\widehat{\Theta}^{k}(\widetilde{z})} \widetilde{e}_{k}+\overline{\widehat{\Theta}^{n+1}(\widetilde{z})} \widetilde{e}_{n+1} .
\end{aligned}
$$

Lemma 12. For $k=1, \ldots, q$,

$$
\widehat{\psi}^{k}(\widetilde{y}, \widetilde{z})=\sum_{s=1}^{q} C^{k}{ }_{s} \widetilde{y}^{s}+\widehat{\Pi}^{k}(\widetilde{z}),
$$


where $C^{k}{ }_{s}, s=1, \ldots, n-2 q$, are complex numbers, and $\widehat{\Pi}^{k}$ is a holomorphic function.

Proof. We now use the real representation of $\widehat{A}_{5} \circ f$ and $\widehat{A}_{5} \circ \xi$, setting $\widehat{\psi}^{l}=\check{\psi}^{2 l-1}+i \check{\psi}^{2 l}$ for $l=1, \ldots, q, \widehat{\mathcal{F}}=\breve{\mathcal{F}}^{1}+i \breve{\mathcal{F}}^{2}$, and $\widehat{\Theta}^{s}=\check{\Theta}^{2 s-1}+i \check{\Theta}^{2 s}$ : $\check{A}_{5} \circ f \circ \check{\phi}_{4}^{-1}\left(x^{1}, \ldots, x^{2 q}, y^{1}, \ldots, y^{2 n-4 q}, z^{1}, \ldots, z^{2 q}\right)$

$$
=\left(x^{1}, \ldots, x^{2 q}, y^{1}, \ldots, y^{2 n-4 q}, \check{\psi}^{1}(y, z), \ldots, \check{\psi}^{2 q}(y, z), \check{\mathcal{F}}^{1}(y, z), \check{\mathcal{F}}^{2}(y, z)\right),
$$

$\check{A}_{5} \circ \xi \circ \check{\phi}_{4}^{-1}\left(x^{1}, \ldots, x^{2 q}, y^{1}, \ldots, y^{2 n-4 q}, z^{1}, \ldots, z^{2 q}\right)$

$$
=\sum_{k=1}^{q}\left[\check{\Theta}^{2 k-1}(z) e_{2 k-1}-\check{\Theta}^{2 k}(z) e_{2 k}\right]+\check{\Theta}^{2 n+1}(z) e_{2 n+1}-\check{\Theta}^{2 n+2}(z) e_{2 n+2} \text {. }
$$

At any point $m^{\prime}$ of the domain $\check{U}$ of $\check{\phi}_{4}$, ker $S$ is spanned by

$$
\frac{\partial}{\partial x^{1}}, \ldots, \frac{\partial}{\partial x^{2 q}}, \frac{\partial}{\partial y^{1}}, \ldots, \frac{\partial}{\partial y^{2 n-4 q}} .
$$

For any $W \in T_{m^{\prime}} M$ and any $j=1, \ldots, 2 n-4 q$,

$$
S\left(\nabla_{W} \frac{\partial}{\partial y^{j}}\right)=\nabla_{W}\left(S \frac{\partial}{\partial y^{j}}\right)=0,
$$

therefore

$$
\begin{aligned}
& \nabla_{\frac{\partial}{\partial y^{s}}} \frac{\partial}{\partial y^{j}}=\sum_{k=1}^{2 q} \alpha_{s j}^{k} \frac{\partial}{\partial x^{k}}+\sum_{l=1}^{2 n-4 q} \beta_{s j}^{l} \frac{\partial}{\partial y^{l}} \\
& \nabla_{\frac{\partial}{\partial z^{s}}} \frac{\partial}{\partial y^{j}}=\sum_{k=1}^{2 q} \gamma_{s j}^{k} \frac{\partial}{\partial x^{k}}+\sum_{l=1}^{2 n-4 q} \delta_{s j}^{l} \frac{\partial}{\partial y^{l}} .
\end{aligned}
$$

We have

$$
\begin{aligned}
\left(\check{A}_{5} \circ f\right)_{*}\left(\frac{\partial}{\partial x^{k}}\right) & =e_{k}, \\
\left(\check{A}_{5} \circ f\right)_{*}\left(\frac{\partial}{\partial y^{l}}\right) & =e_{2 q+l}+\sum_{r=1}^{2 q} \frac{\partial \check{\psi}^{r}}{\partial y^{l}} e_{2 n-2 q+r}+\frac{\partial \check{\mathcal{F}}^{1}}{\partial y^{l}} e_{2 n+1}+\frac{\partial \check{\mathcal{F}}^{2}}{\partial y^{l}} e_{2 n+2} ;
\end{aligned}
$$

hence

$$
\begin{aligned}
\left(\check{A}_{5} \circ f\right)_{*} & \left(\nabla_{\partial / \partial y^{s}} \frac{\partial}{\partial y^{j}}\right) \\
= & \sum_{k=1}^{2 q} \alpha_{s j}^{k} e_{k}+\sum_{l=1}^{2 n-4 q} \beta_{s j}^{l} e_{2 q+l}+\sum_{r=1}^{2 q}\left(\sum_{l=1}^{2 n-4 q} \beta_{s j}^{l} \frac{\partial \check{\psi}^{r}}{\partial y^{l}}\right) e_{2 n-2 q+r} \\
& +\left(\sum_{l=1}^{2 n-4 q} \beta_{s j}^{l} \frac{\partial \check{\mathcal{F}}^{1}}{\partial y^{l}}\right) e_{2 n+1}+\left(\sum_{l=1}^{2 n-4 q} \beta_{s j}^{l} \frac{\partial \check{\mathcal{F}}^{2}}{\partial y^{l}}\right) e_{2 n+2}
\end{aligned}
$$


and

$$
\begin{aligned}
\left(\check{A}_{5} \circ f\right)_{*} & \left(\nabla_{\partial / \partial z^{s}} \frac{\partial}{\partial y^{j}}\right) \\
= & \sum_{k=1}^{2 q} \gamma_{s j}^{k} e_{k}+\sum_{l=1}^{2 n-4 q} \delta_{s j}^{l} e_{2 q+l}+\sum_{r=1}^{2 q}\left(\sum_{l=1}^{2 n-4 q} \delta_{s j}^{l} \frac{\partial \check{\psi}^{r}}{\partial y^{l}}\right) e_{2 n-2 q+r} \\
& +\left(\sum_{l=1}^{2 n-4 q} \delta_{s j}^{l} \frac{\partial \check{\mathcal{F}}^{1}}{\partial y^{l}}\right) e_{2 n+1}+\left(\sum_{l=1}^{2 n-4 q} \delta_{s j}^{l} \frac{\partial \check{\mathcal{F}}^{2}}{\partial y^{l}}\right) e_{2 n+2} .
\end{aligned}
$$

On the other hand, using the Gauss formula we may write

$$
\begin{aligned}
& \left(\check{A}_{5} \circ f\right)_{*}\left(\nabla_{\partial / \partial y^{s}} \frac{\partial}{\partial y^{j}}\right) \\
& =D_{\partial / \partial y^{s}}\left(\check{A}_{5} \circ f\right)_{*} \frac{\partial}{\partial y^{j}}-h\left(\frac{\partial}{\partial y^{s}}, \frac{\partial}{\partial y^{j}}\right) \check{A}_{5} \xi+h\left(J \frac{\partial}{\partial y^{s}}, \frac{\partial}{\partial y^{j}}\right) J \check{A}_{5} \xi \\
& =\sum_{k=1}^{q}\left[\left(-h\left(\frac{\partial}{\partial y^{s}}, \frac{\partial}{\partial y^{j}}\right) \check{\Theta}^{2 k-1}(z)+h\left(J \frac{\partial}{\partial y^{s}}, \frac{\partial}{\partial y^{j}}\right) \check{\Theta}^{2 k}(z)\right) e_{2 k-1}\right. \\
& \left.+\left(h\left(\frac{\partial}{\partial y^{s}}, \frac{\partial}{\partial y^{j}}\right) \check{\Theta}^{2 k}(z)+h\left(J \frac{\partial}{\partial y^{s}}, \frac{\partial}{\partial y^{j}}\right) \check{\Theta}^{2 k-1}(z)\right) e_{2 k}\right] \\
& +\sum_{r=1}^{2 q} \frac{\partial^{2} \check{\psi}^{r}}{\partial y^{s} \partial y^{j}} e_{2 n-2 q+r} \\
& +\left(\frac{\partial^{2} \check{\mathcal{F}}^{1}}{\partial y^{s} \partial y^{j}}-h\left(\frac{\partial}{\partial y^{s}}, \frac{\partial}{\partial y^{j}}\right) \check{\Theta}^{2 n+1}(z)+h\left(J \frac{\partial}{\partial y^{s}}, \frac{\partial}{\partial y^{j}}\right) \check{\Theta}^{2 n+2}(z)\right) e_{2 n+1} \\
& +\left(\frac{\partial^{2} \check{\mathcal{F}}^{2}}{\partial y^{s} \partial y^{j}}+h\left(\frac{\partial}{\partial y^{s}}, \frac{\partial}{\partial y^{j}}\right) \check{\Theta}^{2 n+2}(z)+h\left(J \frac{\partial}{\partial y^{s}}, \frac{\partial}{\partial y^{j}}\right) \check{\Theta}^{2 n+1}(z)\right) e_{2 n+2}
\end{aligned}
$$

and similarly

$$
\begin{aligned}
\left(\check{A}_{5} \circ f\right)_{*} & \left(\nabla_{\partial / \partial z^{s}} \frac{\partial}{\partial y^{j}}\right) \\
= & D_{\partial / \partial z^{s}}\left(\check{A}_{5} \circ f\right)_{*} \frac{\partial}{\partial y^{j}}-h\left(\frac{\partial}{\partial z^{s}}, \frac{\partial}{\partial y^{j}}\right) \check{A}_{5} \xi+h\left(J \frac{\partial}{\partial z^{s}}, \frac{\partial}{\partial y^{j}}\right) J \check{A}_{5} \xi \\
= & \sum_{k=1}^{q}\left[\left(-h\left(\frac{\partial}{\partial z^{s}}, \frac{\partial}{\partial y^{j}}\right) \check{\Theta}^{2 k-1}(z)+h\left(J \frac{\partial}{\partial z^{s}}, \frac{\partial}{\partial y^{j}}\right) \check{\Theta}^{2 k}(z)\right) e_{2 k-1}\right. \\
& \left.+\left(h\left(\frac{\partial}{\partial z^{s}}, \frac{\partial}{\partial y^{j}}\right) \check{\Theta}^{2 k}(z)+h\left(J \frac{\partial}{\partial z^{s}}, \frac{\partial}{\partial y^{j}}\right) \check{\Theta}^{2 k-1}(z)\right) e_{2 k}\right]
\end{aligned}
$$




$$
\begin{aligned}
& +\sum_{r=1}^{2 q} \frac{\partial^{2} \check{\psi}^{r}}{\partial z^{s} \partial y^{j}} e_{2 n-2 q+r} \\
& +\left(\frac{\partial^{2} \check{\mathcal{F}}^{1}}{\partial z^{s} \partial y^{j}}-h\left(\frac{\partial}{\partial z^{s}}, \frac{\partial}{\partial y^{j}}\right) \check{\Theta}^{2 n+1}(z)+h\left(J \frac{\partial}{\partial z^{s}}, \frac{\partial}{\partial y^{j}}\right) \check{\Theta}^{2 n+2}(z)\right) e_{2 n+1} \\
& +\left(\frac{\partial^{2} \check{\mathcal{F}}^{2}}{\partial z^{s} \partial y^{j}}+h\left(\frac{\partial}{\partial z^{s}}, \frac{\partial}{\partial y^{j}}\right) \check{\Theta}^{2 n+2}(z)+h\left(J \frac{\partial}{\partial z^{s}}, \frac{\partial}{\partial y^{j}}\right) \check{\Theta}^{2 n+1}(z)\right) e_{2 n+2} .
\end{aligned}
$$

Since in the second pair of expressions there are no terms containing the basis vectors $e_{t}$ with $t \in\{2 q+1, \ldots, 2 n-2 q\}$, we conclude that $\beta_{s j}^{l}=0$ and $\delta_{s j}^{l}=0$ for any $l, s, j$. Comparing now the coefficients of $e_{t^{\prime}}$ with $t^{\prime} \in$ $\{2 n-2 q+1, \ldots, 2 n\}$ we obtain

$$
\frac{\partial^{2} \check{\psi}^{r}}{\partial y^{s} \partial y^{j}}=0 \quad \text { and } \quad \frac{\partial^{2} \check{\psi}^{r}}{\partial z^{s} \partial y^{j}}=0
$$

for any $r, s, j$.

It follows that

$$
\frac{\partial \check{\psi}^{r}}{\partial y^{j}}=E_{j}^{r}=\mathrm{const} \quad \text { and } \quad \check{\psi}^{r}(y, z)=\sum_{j=1}^{2 n-4 q} E_{j}^{r} y^{j}+\check{\Pi}^{r}(z) .
$$

The Cauchy-Riemann equations for $\widehat{\psi}^{r}$ imply that $\widehat{\Pi}^{r}:=\check{\Pi}^{2 r-1}+i \check{\Pi}^{2 r}$ is a holomorphic function and $E_{2 j-1}^{2 r-1}=E_{2 j}^{2 r}, E_{2 j}^{2 r-1}=-E_{2 j-1}^{2 r}$ for $r=1, \ldots, q$, $j=1, \ldots, n-2 q$. We set $C_{s}^{k}:=E_{2 j-1}^{2 k-1}+i E_{2 j-1}^{2 k}$ and the lemma follows.

We define the isomorphism $\widehat{A}_{6}^{0}$ of $\mathbb{C}^{n+1}$ by

$$
\widehat{A}_{6}^{0} \widetilde{e}_{k}:= \begin{cases}\widetilde{e}_{k} & \text { if } k \notin\{q+1, \ldots, n-q\}, \\ \widetilde{e}_{k}-\sum_{j=1}^{q} C^{j}{ }_{k} \widetilde{e}_{n-q+j} & \text { if } k \in\{q+1, \ldots, n-q\} .\end{cases}
$$

Let $\widehat{A}_{6}:=\widehat{A}_{6}^{0} \circ \widehat{A}_{5}$. Then

$$
\begin{aligned}
\widehat{A}_{6} \circ f \circ \widehat{\phi}_{4}^{-1}\left(\widetilde{x}^{1}, \ldots, \widetilde{x}^{q}, \widetilde{y}^{1}, \ldots, \widetilde{y}^{n-2 q}, \widetilde{z}^{1}, \ldots, \widetilde{z}^{q}\right) \\
\quad=\left(\widetilde{x}^{1}, \ldots, \widetilde{x}^{q}, \widetilde{y}^{1}, \ldots, \widetilde{y}^{n-2 q}, \widehat{\Pi}^{1}(\widetilde{z}), \ldots, \widehat{\Pi}^{q}(\widetilde{z}), \widehat{\mathcal{F}}(\widetilde{y}, \widetilde{z})\right)
\end{aligned}
$$

and $\widehat{A}_{6} \xi \circ \widehat{\phi}_{4}^{-1}$ is given by the same formula as $\widehat{A}_{5} \xi \circ \widehat{\phi}_{4}^{-1}$.

Lemma 13. If $q>1$ then

$$
\widehat{\Pi}^{r}(\widetilde{z})=\sum_{s=1}^{q} C_{1}^{r}{ }_{s} \widetilde{z}^{s}+C_{2}^{r}
$$

with $C_{1}{ }^{r}{ }_{s}, C_{2}{ }^{r} \in \mathbb{C}$. 
Proof. Recall that for $q>1$,

$$
\begin{aligned}
\widehat{\Theta}^{k}\left(\widetilde{z}^{1}, \ldots, \widetilde{z}^{q}\right) & =\widetilde{z}^{k} \quad \text { for } k=1, \ldots, q, \\
\widehat{\Theta}^{n+1}\left(\widetilde{z}^{1}, \ldots, \widetilde{z}^{q}\right) & \equiv 1 .
\end{aligned}
$$

Therefore we have

$$
\begin{aligned}
A_{6} \xi \circ \phi_{4}^{-1}\left(x^{1}, \ldots, x^{2 q}, y^{1}, \ldots, y^{2 n-4 q}, z^{1}, \ldots, z^{2 q}\right) & \\
& =\sum_{k=1}^{q}\left[z^{2 k-1} e_{2 k-1}-z^{2 k} e_{2 k}\right]+e_{2 n+1},
\end{aligned}
$$

and

$$
D_{\partial / \partial z^{s}}\left(A_{6} \xi\right)=(-1)^{s-1} e_{s}=-\left(A_{6} \circ f\right)_{*}\left((-1)^{s} \frac{\partial}{\partial x^{s}}\right),
$$

which implies

$$
S \frac{\partial}{\partial z^{s}}=(-1)^{s} \frac{\partial}{\partial x^{s}} .
$$

Applying the covariant derivative $\nabla_{W}$ to the right-hand side of the above formula we obtain zero, therefore

$$
S\left(\nabla_{W} \frac{\partial}{\partial z^{s}}\right)=\nabla_{W}\left(S \frac{\partial}{\partial z^{s}}\right)=0
$$

and so $\nabla_{W}\left(\partial / \partial z^{s}\right) \in \operatorname{ker} S$ for any tangent vector $W$. We can now proceed analogously to the proof of Lemma 12 .

The matrix $\left(C_{1}{ }^{r}{ }_{s}\right)_{r, s=1, \ldots, q}$ is invertible, since $\widehat{A}_{6} \circ f$ is an immersion and

$$
\widehat{A}_{6} \xi \circ \widehat{\phi}_{4}^{-1}\left(\widetilde{x}^{1}, \ldots, \widetilde{x}^{q}, \widetilde{y}^{1}, \ldots, \widetilde{y}^{n-2 q}, \widetilde{z}^{1}, \ldots, \widetilde{z}^{q}\right)=\sum_{k=1}^{q} \overline{\widetilde{z}^{k}} \widetilde{e}_{k}+\widetilde{e}_{n+1}
$$

is a transversal field. Let

$$
\left(C_{3}{ }^{i}{ }_{j}\right)_{i, j=1, \ldots, q}=\left[\left(C_{1}{ }^{r}{ }_{s}\right)_{r, s=1, \ldots, q}\right]^{-1} .
$$

To complete the proof of the theorem in the case $q>1$ it remains to apply the affine isomorphism $\widetilde{A}_{7}^{0}$ of $\mathbb{C}^{n+1}$, where

$$
\begin{aligned}
\widetilde{A}_{7}^{0}\left(\theta^{1}, \ldots, \theta^{n+1}\right):=\left(\theta^{1}, \ldots, \theta^{n-q}, \sum_{j_{1}=1}^{q} C_{3}{ }^{1}{ }_{j_{1}}\left(\theta^{n-q+j_{1}}-C_{2}{ }^{j_{1}}\right), \ldots,\right. \\
\\
\left.\sum_{j_{q}=1}^{q} C_{3}{ }^{q}{ }_{j_{q}}\left(\theta^{n-q+j_{q}}-C_{2}{ }^{j_{q}}\right), \theta^{n+1}\right) .
\end{aligned}
$$

The linear part of $\widetilde{A}_{7}^{0}$ does not change the transversal field $\widehat{A}_{6} \xi$, therefore we have $\widetilde{A} \circ f$ and $\overrightarrow{\widetilde{A}} \xi$ as claimed, with $\widetilde{A}:=\widetilde{A}_{7}^{0} \circ \widehat{A}_{6}, \widetilde{\mathcal{F}}=\widehat{\mathcal{F}}, \widetilde{\phi}=\widehat{\phi}_{4}$. 
For $q=1$,

$$
\widehat{A}_{6} \circ f \circ \widehat{\phi}_{4}^{-1}\left(\widetilde{x}, \widetilde{y}^{1}, \ldots, \widetilde{y}^{n-2}, \widetilde{z}\right)=\left(\widetilde{x}, \widetilde{y}^{1}, \ldots, \widetilde{y}^{n-2}, \widehat{\Pi}(\widetilde{z}), \widehat{\mathcal{F}}(\widetilde{y}, \widetilde{z})\right)
$$

with $\widehat{\Pi}^{\prime} \neq 0$ in the neighbourhood of $\widetilde{\widetilde{\phi}}_{4}(m)$. We define a local diffeomorphism

$$
\widetilde{\widetilde{\phi}}_{5}^{0}\left(\widetilde{x}, \widetilde{y}^{1}, \ldots, \widetilde{y}^{n-2}, \widetilde{z}\right):=\left(\widetilde{x}, \widetilde{y}^{1}, \ldots, \widetilde{y}^{n-2}, \widehat{\Pi}(\widetilde{z})\right)
$$

and obtain

$$
\begin{aligned}
\widehat{A}_{6} \circ f \circ \widetilde{\widetilde{\phi}}_{5}^{-1}\left(\widetilde{x}, \widetilde{y}^{1}, \ldots, \widetilde{y}^{n-2}, \widetilde{z}\right) & =\left(\widetilde{x}, \widetilde{y}^{1}, \ldots, \widetilde{y}^{n-2}, \widetilde{z}, \widehat{\mathcal{\mathcal { F }}}(\widetilde{y}, \widetilde{z})\right), \\
\widehat{A}_{6} \xi \circ \widetilde{\widetilde{\phi}}_{5}^{-1}\left(\widetilde{x}, \widetilde{y}^{1}, \ldots, \widetilde{y}^{n-2}, \widetilde{z}\right) & =\overline{\widetilde{\mathcal{G}}(\widetilde{z})} \widetilde{e}_{1}+\overline{e^{\widetilde{\mathcal{M}}}(\widetilde{z})} \widetilde{e}_{n+1}
\end{aligned}
$$

with $\widehat{\widehat{\mathcal{F}}}=\widehat{\mathcal{F}} \circ \widehat{\Pi}^{-1}, \widetilde{\widetilde{\mathcal{M}}}=\widetilde{\mathcal{H}} \circ \widehat{\Pi}^{-1}, \widetilde{\widetilde{\mathcal{G}}}=\Theta^{1} \circ \widehat{\Pi}^{-1}, \widetilde{\bar{\phi}}_{5}=\widetilde{\widetilde{\phi}}_{5}^{0} \circ \widehat{\phi}_{4}$.

Lemma 14. $\widetilde{\widetilde{\mathcal{G}}^{\prime}}-\widetilde{\widetilde{\mathcal{M}}^{\prime}} \widetilde{\widetilde{\mathcal{G}}}=$ const $\neq 0$.

Proof. Let $\widetilde{\widetilde{\mathcal{G}}}=G^{1}+i G^{2}$ and $\widetilde{\widetilde{\mathcal{M}}}=M^{1}+i M^{2}$. Then

$$
\begin{aligned}
& \widetilde{\widetilde{\mathcal{G}}^{\prime}}-\widetilde{\widetilde{\mathcal{M}}^{\prime}} \widetilde{\widetilde{\mathcal{G}}} \\
& =\left(\frac{\partial G^{1}}{\partial z^{1}}+i \frac{\partial G^{2}}{\partial z^{1}}\right)-\left(\frac{\partial M^{1}}{\partial z^{1}}+i \frac{\partial M^{2}}{\partial z^{1}}\right)\left(G^{1}+i G^{2}\right) \\
& =\left(\frac{\partial G^{1}}{\partial z^{1}}-\frac{\partial M^{1}}{\partial z^{1}} G^{1}+\frac{\partial M^{2}}{\partial z^{1}} G^{2}\right)+i\left(\frac{\partial G^{2}}{\partial z^{1}}-\frac{\partial M^{2}}{\partial z^{1}} G^{1}-\frac{\partial M^{1}}{\partial z^{1}} G^{2}\right) .
\end{aligned}
$$

It is easily seen that

$$
\begin{aligned}
S \frac{\partial}{\partial z^{1}}= & \left(-\frac{\partial G^{1}}{\partial z^{1}}+\frac{\partial M^{1}}{\partial z^{1}} G^{1}-\frac{\partial M^{2}}{\partial z^{1}} G^{2}\right) \frac{\partial}{\partial x^{1}} \\
& +\left(\frac{\partial G^{2}}{\partial z^{1}}-\frac{\partial M^{2}}{\partial z^{1}} G^{1}-\frac{\partial M^{1}}{\partial z^{1}} G^{2}\right) \frac{\partial}{\partial x^{2}}
\end{aligned}
$$

because

$$
\begin{aligned}
D_{\partial / \partial z^{1}} A_{6} \xi= & \frac{\partial G^{1}}{\partial z^{1}} e_{1}-\frac{\partial G^{2}}{\partial z^{1}} e_{2} \\
& +\left(\frac{\partial M^{1}}{\partial z^{1}} e^{M^{1}} \cos M^{2}-\frac{\partial M^{2}}{\partial z^{1}} e^{M^{1}} \sin M^{2}\right) e_{2 n+1} \\
& +\left(-\frac{\partial M^{1}}{\partial z^{1}} e^{M^{1}} \sin M^{2}-\frac{\partial M^{2}}{\partial z^{1}} e^{M^{1}} \cos M^{2}\right) e_{2 n+2} \\
= & \left(\frac{\partial G^{1}}{\partial z^{1}}-\frac{\partial M^{1}}{\partial z^{1}} G^{1}+\frac{\partial M^{2}}{\partial z^{1}} G^{2}\right) e_{1} \\
& +\left(-\frac{\partial G^{2}}{\partial z^{1}}+\frac{\partial M^{2}}{\partial z^{1}} G^{1}+\frac{\partial M^{1}}{\partial z^{1}} G^{2}\right) e_{2}+\frac{\partial M^{1}}{\partial z^{1}} A_{6} \xi-\frac{\partial M^{2}}{\partial z^{1}} J A_{6} \xi
\end{aligned}
$$


For any vector $W$,

$$
\nabla_{W} \frac{\partial}{\partial z^{1}} \in \operatorname{span}_{\mathbb{R}}\left\{\frac{\partial}{\partial x^{1}}, \frac{\partial}{\partial x^{2}}\right\} \subset \operatorname{ker} S,
$$

since

$$
\begin{aligned}
D_{W}\left(A_{6} \circ f\right)_{*} \frac{\partial}{\partial z^{1}} & =W\left(\frac{\partial \check{\mathcal{F}}^{1}}{\partial z^{1}}\right) e_{2 n+1}+W\left(\frac{\partial \check{\mathcal{F}}^{2}}{\partial z^{1}}\right) e_{2 n+2} \\
& \in \operatorname{span}_{\mathbb{R}}\left\{e_{1}, e_{2}, A_{6} \xi, J A_{6} \xi\right\} .
\end{aligned}
$$

It follows that $\nabla_{W}\left(S \partial / \partial z^{1}\right)=0$ for any $W$. Therefore

$$
\begin{gathered}
-\frac{\partial G^{1}}{\partial z^{1}}+\frac{\partial M^{1}}{\partial z^{1}} G^{1}-\frac{\partial M^{2}}{\partial z^{1}} G^{2}=:-B_{1}=\text { const, } \\
\frac{\partial G^{2}}{\partial z^{1}}-\frac{\partial M^{2}}{\partial z^{1}} G^{1}-\frac{\partial M^{1}}{\partial z^{1}} G^{2}=: B_{2}=\text { const. }
\end{gathered}
$$

Moreover, $\left(B_{1}\right)^{2}+\left(B_{2}\right)^{2} \neq 0$, because $S \neq 0$.

Let

$$
\begin{aligned}
\widetilde{\widetilde{\phi}}_{6}^{0}\left(\widetilde{x}, \widetilde{y}^{1}, \ldots, \widetilde{y}^{n-2}, \widetilde{z}\right) & :=\left(\widetilde{x}, \widetilde{y}^{1}, \ldots, \widetilde{y}^{n-2},\left(B_{1}+i B_{2}\right) \widetilde{z}\right), \\
\widetilde{\widetilde{A}}_{7}^{0}\left(\zeta^{1}, \ldots, \zeta^{n+1}\right) & :=\left(\zeta^{1}, \ldots, \zeta^{n-1},\left(B_{1}+i B_{2}\right) \zeta^{n}, \zeta^{n+1}\right) .
\end{aligned}
$$

Now $\widetilde{\widetilde{A}}_{7} \circ f \circ \widetilde{\widetilde{\phi}}_{6}^{-1}$ and $\widetilde{\widetilde{A}}_{7} \xi \circ \widetilde{\widetilde{\phi}}_{6}^{-1}$ have the same shape as $\widehat{A}_{6} \circ f \circ \widetilde{\widetilde{\phi}}_{5}^{-1}$ and $\widehat{A}_{6} \xi \circ \widetilde{\widetilde{\phi}}_{5}^{-1}$ with $\widetilde{\widetilde{\mathcal{F}}}, \widetilde{\widetilde{\mathcal{G}}}, \widetilde{\widetilde{\mathcal{M}}}$ replaced by $\widetilde{\mathcal{F}}, \widetilde{\mathcal{G}} \widetilde{\mathcal{M}}$, where

$$
\begin{gathered}
\widetilde{\mathcal{F}}(\widetilde{z}):=\widehat{\mathcal{F}}\left(\frac{\widetilde{z}}{B_{1}+i B_{2}}\right), \quad \widetilde{\mathcal{G}}(\widetilde{z}):=\widetilde{\widetilde{\mathcal{G}}}\left(\frac{\widetilde{z}}{B_{1}+i B_{2}}\right), \\
\widetilde{\mathcal{M}}(\widetilde{z}):=\widetilde{\mathcal{M}}\left(\frac{\widetilde{z}}{B_{1}+i B_{2}}\right) .
\end{gathered}
$$

It is easy to check that

$$
\widetilde{\mathcal{G}}^{\prime}-\widetilde{\mathcal{M}^{\prime}} \widetilde{\mathcal{G}}=1 .
$$

This finishes the proof of the theorem.

There are many examples of functions $\widetilde{\mathcal{G}}$ and $\widetilde{\mathcal{M}}$ satisfying the above equation. In fact, for any holomorphic $\widetilde{\mathcal{M}}$ (and so for any $\mu, \nu$, because $\mu\left(\partial / \partial z^{k}\right)=\partial \mathcal{M}^{1} / \partial z^{k}$ and $\left.\nu\left(\partial / \partial z^{k}\right)=-\partial \mathcal{M}^{1} / \partial z^{k}\right)$ there exists $\widetilde{\mathcal{G}}$ such that $\widetilde{\mathcal{G}}^{\prime}-\widetilde{\mathcal{M}}^{\prime} \widetilde{\mathcal{G}} \equiv 1$. For example

$$
\widetilde{\mathcal{G}}(\widetilde{z})=\widetilde{z}, \quad \widetilde{\mathcal{M}}(\widetilde{z})=0
$$

(that is what we obtain also for $q>1$, with $\mu=\nu=0$ );

$$
\begin{array}{ll}
\widetilde{\mathcal{G}}(\widetilde{z})=1, & \widetilde{\mathcal{M}}(\widetilde{z})=-\widetilde{z} ; \\
\widetilde{\mathcal{G}}(\widetilde{z})=e^{\widetilde{z}}, & \widetilde{\mathcal{M}}(\widetilde{z})=\widetilde{z}+e^{-\widetilde{z}} .
\end{array}
$$




\section{References}

[DVV] F. Dillen, L. Vrancken and L. Verstraelen, Complex affine differential geometry, Atti. Acad. Peloritana Pericolanti Cl. Sci. Fis. Mat. Nat. 66 (1988), 231-260.

[NS] K. Nomizu and T. Sasaki, Affine Differential Geometry, Cambridge Univ. Press, 1994.

[O] B. Opozda, Equivalence theorems for complex affine hypersurfaces, Results Math. 27 (1995), 316-327.

Institute of Mathematics

Jagiellonian University

Reymonta 4

30-059 Kraków, Poland

E-mail: robaszew@im.uj.edu.pl 